\title{
Robust Iterative Learning Control for 2-D Singular Fornasini-Marchesini Systems with Iteration-Varying Boundary States
}

\author{
Deming $X u$ and Kai Wan \\ School of Electronic Information and Electrical Engineering, Huizhou University, Huizhou 516007, China \\ Correspondence should be addressed to Kai Wan; wankai606815@163.com
}

Received 3 November 2020; Revised 7 February 2021; Accepted 22 February 2021; Published 1 March 2021

Academic Editor: Heng Liu

Copyright (C) 2021 Deming Xu and Kai Wan. This is an open access article distributed under the Creative Commons Attribution License, which permits unrestricted use, distribution, and reproduction in any medium, provided the original work is properly cited.

\begin{abstract}
This study first investigates robust iterative learning control (ILC) issue for a class of two-dimensional linear discrete singular Fornasini-Marchesini systems (2-D LDSFM) under iteration-varying boundary states. Initially, using the singular value decomposition theory, an equivalent dynamical decomposition form of 2-D LDSFM is derived. A simple P-type ILC law is proposed such that the ILC tracking error can be driven into a residual range, the bound of which is relevant to the bound parameters of boundary states. Specially, while the boundary states of 2-D LDSFM satisfy iteration-invariant boundary states, accurate tracking on 2-D desired surface trajectory can be accomplished by using 2-D linear inequality theory. In addition, extension to 2-D LDSFM without direct transmission from inputs to outputs is presented. A numerical example is used to illustrate the effectiveness and feasibility of the designed ILC law.
\end{abstract}

\section{Introduction}

Two-dimensional (2-D) singular dynamical systems derived from the discretization of spatiotemporal dynamical systems with singular matrices or singular distributed parameter systems have received much attention due to their extensive applications in physical phenomena and industrial processes, such as electrical circuits [1], nanoelectronics [2], transmission lines in signal propagation [3], and power systems [4]. In recent years, fruitful results on 2-D singular systems in the infinite coordinate domain have been reported, mainly including the detectability and observer design [5], $H_{\infty}$ control [6], and stability analysis [7]. However, in practical industrial applications, 2-D singular systems are often required to execute some specific tracking control tasks repetitively over the finite coordinate domain. For example, in form-closure grasps, the immobilized manipulation of serial chains described by 2 -D singular systems could be regarded as a repetitive control problem [8]. Also, in the field of mold processing and material surface manufacturing, it is usually required to obtain high-precision 2-D reference surface by repeated operations of the controlled processing units [9]. Obviously, for the repetitively tracking cases mentioned above, the traditional tracking control approaches for 2-D singular systems in the infinite coordinate domain is difficult to be applicable.

Iterative learning control (ILC), as an intelligent control method, is used to address the repetitive trajectory tracking problem for one-dimensional (1-D) dynamical systems with less precise model knowledge, which makes ILC be widely prevalent in practical applications. A large number of ILC research results for 1-D dynamical systems have been reported in the past decades [10-16]. However, only very few ILC results for $2-\mathrm{D}$ dynamical systems is involved in [17-24]. For 2-D linear discrete nonsingular Fornasini-Marchesini systems (2-D LDNFM), a two-gain ILC algorithm is proposed in [20] to address the robust ILC issue under the iteration-varying boundary states, and the detailed proof process of convergence analysis is given. Also, to track a class of nonrepetitive reference 
surface trajectory represented by a high-order internal model (HOIM) operator, two HOIM-based ILC laws were, respectively, investigated in [21] for 2-D LDNFM by using HOIM-based linear inequality theory, but the ultimate ILC tracking error can only converge to a bounded range. To accomplish the objective of zero tracking error, an adaptive ILC technique is proposed in [24] to deal with the tracking problem of iteration-varying reference surface trajectory. Unfortunately, it requires that the gain matrix of 2-D LDNFM must be positive-definite (or negative-definite), such that the proposed adaptive ILC algorithm, in practical applications, are severely confined. It is worth emphasizing that the aforementioned ILC results have concerned on a nonsingular case. However, in practical life and industry, there exist some 2-D singular dynamical systems, such as electrical circuits, transmission lines in signal propagation, and power systems, which are often required to execute some specific tracking control tasks repetitively. Based on these practical applications, it is essential to exploit ILC techniques for 2-D singular dynamical systems.

The main aim of this paper is to investigate the robustness and convergence property of P-type ILC law for two classes of two-dimensional linear discrete singular Fornasini-Marchesini systems (2-D LDSFM) under iteration-varying boundary states. By using singular value decomposition theory, an equivalent dynamical decomposition form of 2-D LDSFM is derived. By using 2-D linear inequality theory, it can guarantee that the ultimate tracking error tends to a bounded range, the bound of which is relevant to the bound parameters of boundary states. The main contributions of this paper relative to the related works are summarized as follows:

(1) In the existing ILC results for 2-D linear discrete systems [17-24], they are concerned on a nonsingular case. To the best of our knowledge, this is the first time to investigate robust ILC algorithms for 2D discrete singular systems in this paper.

(2) Compared with the adaptive ILC algorithm for 2-D LDNFM in [24], the ILC algorithm proposed in this paper has no restriction on the numbers of system inputs and outputs.

(3) In the study of ILC algorithms for 1-D singular systems [25-27], the $\lambda$-norm and identical boundary condition have been widely used to analysis the convergence of the proposed ILC laws. However, the $\lambda$-norm might not provide a satisfactory measurement of ILC tracking errors [22], and thus, it is not involved in analysing the proposed ILC law in the paper.

The remaining section of this paper is arranged as follows: The problem description is provided in Section 2. Sections 3 and 4, respectively, present robustness and convergence analysis of P-type ILC law for 2-D LDSFM (1) and extension to 2-D LDSFM with $D=0$. In Section 5, a simulation example is introduced. Finally, Section 6 gives a conclusion.

\section{Problem Description}

Consider a ILC issue for the following two-dimensional linear discrete singular Fornasini-Marchesini systems (2-D LDSFM) [28] over finite region $n_{1} \in\left\{0,1, \ldots, N_{1}-1\right\}$ and $n_{2} \in\left\{0,1, \ldots, N_{2}-1\right\}$ :

$$
\left\{\begin{array}{l}
E_{g} x_{k}\left(n_{1}+1, n_{2}+1\right)=A_{1} x_{k}\left(n_{1}+1, n_{2}\right)+A_{2} x_{k}\left(n_{1}, n_{2}\right) \\
+A_{3}\left(n_{1}, n_{2}+1\right)+B u_{k}\left(n_{1}, n_{2}\right) \\
y_{k}\left(n_{1}, n_{2}\right)=C x_{k}\left(n_{1}, n_{2}\right)+D u_{k}\left(n_{1}, n_{2}\right)
\end{array}\right.
$$

where $u_{k}\left(n_{1}, n_{2}\right) \in R^{m}, x_{k}\left(n_{1}, n_{2}\right) \in R^{p}$, and $y_{k}\left(n_{1}, n_{2}\right) \in R^{s}$ represent, respectively, control input, system state, and system output and $E_{g}, A_{1}, A_{2}, A_{3}, B, C$, and $D$ are real matrices with appropriate dimensions. $k \in\{0,1,2, \ldots\}$ denotes the $k$-th iteration of controlled system (1); $n_{1}$ and $n_{2}$ are, respectively, horizontal dynamical index and vertical dynamical index. It is worth noting that as $E_{g}$ is nonsingular (without loss of generality, let $E_{g}=I_{p}$, where $I_{p}$ represents identity matrix with $(p \times p))$, (1) is called a regular $2-\mathrm{D}$ LDFFM [28], which has been investigated in [21]. However, in this paper, a singular matrix $E_{g}$ with $\operatorname{rank}\left(E_{g}\right)=r<p$ is considered.

For $n_{1} \in\left\{0,1, \ldots, N_{1}\right\}$ and $n_{2} \in\left\{0,1, \ldots, N_{2}\right\}$, let an achievable desired surface trajectory and the corresponding tracking error at $k$-th iteration be denoted as $y_{d}\left(n_{1}, n_{2}\right)$ and $e_{k}\left(n_{1}, n_{2}\right)$, respectively. The control objective of ILC for 2-D LDSFM (1) is to generate a control input sequence $u_{k}\left(n_{1}, n_{2}\right)$, where $n_{1} \in\left\{0,1, \ldots, N_{1}-1\right\} \quad$ and $n_{2} \in\left\{0,1, \ldots, N_{2}-1\right\}$ with an iterative way, such that the actual tracking output $y_{k}\left(n_{1}, n_{2}\right)$ can accurately track $y_{d}\left(n_{1}, n_{2}\right)$, i.e.,

$$
\lim _{k \longrightarrow+\infty} y_{k}\left(n_{1}, n_{2}\right)=y_{d}\left(n_{1}, n_{2}\right)
$$

where $n_{1} \in\left\{0,1, \ldots, N_{1}\right\}$ and $n_{2} \in\left\{0,1, \ldots, N_{2}\right\}$.

According to the singular value matrix theory [29], there exist two nonsingular matrices $P \in R^{p \times p}$ and $Q \in R^{p \times p}$ such that

$$
\begin{aligned}
P E_{g} Q & =\left[\begin{array}{ll}
I_{r} & 0 \\
0 & 0
\end{array}\right], \\
P A_{1} Q & =\left[\begin{array}{ll}
\bar{A}_{11} & \bar{A}_{12} \\
\bar{A}_{21} & \bar{A}_{22}
\end{array}\right], \\
P A_{2} Q & =\left[\begin{array}{ll}
\widehat{A}_{11} & \widehat{A}_{12} \\
\widehat{A}_{21} & \widehat{A}_{22}
\end{array}\right], \\
P A_{3} Q & =\left[\begin{array}{ll}
\widetilde{A}_{11} & \widetilde{A}_{12} \\
\widetilde{A}_{21} & \widetilde{A}_{22}
\end{array}\right], \\
P B & =\left[\begin{array}{l}
B_{1} \\
B_{2}
\end{array}\right], \\
C Q & =\left[\begin{array}{ll}
C_{1} & C_{2}
\end{array}\right] .
\end{aligned}
$$

2-D LDSFM (1) can be transformed into a decomposition form 


$$
\begin{aligned}
z_{k}^{1}\left(n_{1}+1, n_{2}+1\right)= & \bar{A}_{11} z_{k}^{1}\left(n_{1}+1, n_{2}\right)+\widetilde{A}_{12} z_{k}^{2}\left(n_{1}+1, n_{2}\right)+\widehat{A}_{11} z_{k}^{1}\left(n_{1}, n_{2}\right)+\widehat{A}_{12} z_{k}^{2}\left(n_{1}, n_{2}\right) \\
& +\widetilde{A}_{11} z_{k}^{1}\left(n_{1}, n_{2}+1\right)+\widetilde{A}_{12} z_{k}^{2}\left(n_{1}, n_{2}+1\right)+B_{1} u_{k}\left(n_{1}, n_{2}\right), \\
0= & \bar{A}_{21} z_{k}^{1}\left(n_{1}+1, n_{2}\right)+\bar{A}_{22} z_{k}^{2}\left(n_{1}+1, n_{2}\right)+\widehat{A}_{21} z_{k}^{1}\left(n_{1}, n_{2}\right)+\widehat{A}_{22} z_{k}^{2}\left(n_{1}, n_{2}\right) \\
& +\widetilde{A}_{21} z_{k}^{1}\left(n_{1}, n_{2}+1\right)+\widetilde{A}_{22} z_{k}^{2}\left(n_{1}, n_{2}+1\right)+B_{2} u_{k}\left(n_{1}, n_{2}\right), \\
y_{k}\left(n_{1}, n_{2}\right)= & C_{1} z_{k}^{1}\left(n_{1}, n_{2}\right)+C_{2} z_{k}^{2}\left(n_{1}, n_{2}\right)+D u_{k}\left(n_{1}, n_{2}\right)
\end{aligned}
$$

where $\quad z_{k}\left(n_{1}, n_{2}\right)=\left[\begin{array}{c}z_{k}^{1}\left(n_{1}, n_{2}\right) \\ z_{k}^{2}\left(n_{1}, n_{2}\right)\end{array}\right]=Q^{-1} x_{k}\left(n_{1}, n_{2}\right)$, $z_{k}^{1}\left(n_{1}, n_{2}\right) \in R^{r}, z_{k}^{2}\left(n_{1}, n_{2}\right) \in R^{p-r}$, and $Q^{-1}=\left[\begin{array}{ll}Q_{11} & Q_{12} \\ Q_{21} & Q_{22}\end{array}\right]$. For some thermal processes in chemical reactors, heat exchangers and pipe furnaces, $z_{k}\left(n_{1}, n_{2}\right)$, usually represents temperature at space $n_{1}$ and time $n_{2}$ in $[6,30]$.

For the convenience of discussing the ILC problem, Definitions 1 and 2 on nonnegative matrix (vector), Assumptions 1-3, and Lemma 1 for 2-D LDSFM (1) are provided.

Definition 1. If every element of a matrix (or vector) is nonnegative, then the matrix (or vector) is said to be nonnegative, i.e., for $A=\left[a_{i j}\right] \in R^{L_{1} \times L_{2}}$, if $a_{i j} \geq 0$, where $i \in\left\{1,2, \ldots, L_{1}\right\}$ and $j \in\left\{1,2, \ldots, L_{2}\right\}$, then it is denoted that $A \geq 0$.

Definition 2. For two matrices $P=\left[p_{i j}\right] \in R^{L_{1} \times L_{2}}$ and $S=\left[s_{i j}\right] \in R^{L_{1} \times L_{2}}, \quad P \leq S \quad$ is denoted if $p_{i j} \leq s_{i j}$ for $i \in\left\{1,2, \ldots, L_{1}\right\}$ and $j \in\left\{1,2, \ldots, L_{2}\right\}$.

Assumption 1. For 2-D LDSFM (1), let boundary states $x_{k}\left(0, n_{2}\right)$ and $x_{k}\left(n_{1}, 0\right)$ be satisfied as $\left\|x_{k}\left(0, n_{2}\right)-x_{0}\left(0, n_{2}\right)\right\| \leq \Lambda_{1}$, with $n_{2} \in\left\{1,2, \cdots, N_{2}\right\}$ and $\left\|x_{k}\left(n_{1}, 0\right)-x_{0}\left(n_{1}, 0\right)\right\| \leq \Lambda_{2}$, with $n_{1} \in\left\{0,1, \cdots, N_{1}\right\}$, where $x_{0}\left(0, n_{2}\right)$ and $x_{0}\left(n_{1}, 0\right)$ are time-varying functions with respective to $n_{2}$ and $n_{1}$, respectively; $\Lambda_{1}$ and $\Lambda_{2}$ are unknown constants. $\|\cdot\|$ represents the infinite norm of vector/matrix in this paper.

Assumption 2. $\widetilde{A}_{22}$ is an invertible matrix.

Assumption 3. 2-D LDSFM (1) is regular, if there exists two complex numbers $z_{1}$ and $z_{2}$ to make $\operatorname{det}\left(E_{g} z_{1} z_{2}-A_{1} z_{1}-A_{2}-A_{3} z_{2}\right) \neq 0$.

Lemma 1. Consider the following 3-D linear discrete inequality system for $n_{1} \in\left\{0,1, \ldots, N_{1}\right\}, n_{2} \in\left\{0,1, \ldots, N_{2}\right\}$, and $k \in\{0,1,2, \ldots\}$ :

$$
\theta_{k+1}\left(n_{1}, n_{2}\right) \leq \alpha \theta_{k}\left(n_{1}, n_{2}\right)+\delta_{k}\left(n_{1}, n_{2}\right),
$$

where $\theta_{k}\left(n_{1}, n_{2}\right) \in R$ and $\delta_{k}\left(n_{1}, n_{2}\right) \in R$, respectively, are state and control input and $\alpha$ denotes real constant. Suppose the boundary state $\theta_{0}\left(n_{1}, n_{2}\right)=f\left(n_{1}, n_{2}\right)$, where $f\left(n_{1}, n_{2}\right)$ is a bounded vector function. When $\limsup \operatorname{su+\infty }_{k} \delta_{k}\left(n_{1}, n_{2}\right) \leq b_{\delta}$, if $0<\alpha<1$ is satisfied, then

$$
\limsup _{k \longrightarrow+\infty} \theta_{k}\left(n_{1}, n_{2}\right) \leq \frac{b_{\delta}}{1-\alpha} .
$$

The proof process of Lemma 1 is similar with [22].

Remark 1. In the existing ILC results for 2-D LDNFM $[20,21,23]$, it is usually required that boundary states are satisfied as identical boundary states. However, in practical ILC applications for 2-D systems, it is difficult to obtain in each repetitive operation. To this end, ILC for 2-D LDSFM (1) under iteration-varying boundary states is investigated in this paper. Assumptions 2 and 3 are basic and reasonable assumptions in control theory of the 2-D singular system $[28,31]$.

\section{Robustness and Convergence Analysis of P-Type ILC Law for 2-D LDSFM (1)}

According to the system characteristic of 2-D LDSFM (1), the following P-type ILC law is proposed for $n_{1} \in\left\{0,1, \ldots, N_{1}\right\}$ and $n_{2} \in\left\{0,1, \ldots, N_{2}\right\}$ :

$$
u_{k+1}\left(n_{1}, n_{2}\right)=u_{k}\left(n_{1}, n_{2}\right)+K e_{k}\left(n_{1}, n_{2}\right),
$$

where the learning gain $K \in R^{m \times s}$ is to be designed.

Theorem 1. For 2-D LDSFM (1) under Assumptions 1-3, use the P-type ILC law (9). If there exists the learning gain $K$ satisfying $\left\|I_{s}-D K\right\|<1$, then the tracking error $e_{k}\left(n_{1}, n_{2}\right)$, $n_{1} \in\left\{0,1, \ldots, N_{1}\right\}, \quad n_{2} \in\left\{0,1, \ldots, N_{2}\right\}$ converges to a bounded range, i.e.,

$$
\underset{k \longrightarrow+\infty}{\limsup }\left\|e_{k}\left(n_{1}, n_{2}\right)\right\| \leq \beta_{e},
$$

where $\beta_{e}$ is a certain bound related to the bound parameters $\Lambda_{1}$ and $\Lambda_{2}$ in Assumption 1.

Proof. For $n_{1} \in\left\{0,1, \ldots, N_{1}\right\}$ and $n_{2} \in\left\{0,1, \ldots, N_{2}\right\}$, let

$$
\begin{aligned}
& \delta z_{k+1}^{1}\left(n_{1}, n_{2}\right)=z_{k+1}^{1}\left(n_{1}, n_{2}\right)-z_{k}^{1}\left(n_{1}, n_{2}\right), \\
& \delta z_{k+1}^{2}\left(n_{1}, n_{2}\right)=z_{k+1}^{2}\left(n_{1}, n_{2}\right)-z_{k}^{2}\left(n_{1}, n_{2}\right) .
\end{aligned}
$$

Using (11) and (5), and considering (4) and (12), there is 


$$
\begin{aligned}
\delta z_{k+1}^{1}\left(n_{1}+1, n_{2}+1\right)= & z_{k+1}^{1}\left(n_{1}+1, n_{2}+1\right)-z_{k}^{1}\left(n_{1}+1, n_{2}+1\right) \\
= & \bar{A}_{11} \delta z_{k+1}^{1}\left(n_{1}+1, n_{2}\right)+\bar{A}_{12} \delta z_{k+1}^{2}\left(n_{1}+1, n_{2}\right)+\widehat{A}_{11} \delta z_{k+1}^{1}\left(n_{1}, n_{2}\right) \\
& +\widehat{A}_{12} \delta z_{k+1}^{2}\left(n_{1}, n_{2}\right)+\widetilde{A}_{11} \delta z_{k+1}^{1}\left(n_{1}, n_{2}+1\right)+\widetilde{A}_{12} \delta z_{k+1}^{2}\left(n_{1}, n_{2}+1\right)+B_{1}\left[u_{k+1}\left(n_{1}, n_{2}\right)-u_{k}\left(n_{1}, n_{2}\right)\right], \\
0= & \bar{A}_{21} \delta z_{k+1}^{1}\left(n_{1}+1, n_{2}\right)+\bar{A}_{22} \delta z_{k+1}^{2}\left(n_{1}+1, n_{2}\right)+\widehat{A}_{21} \delta z_{k+1}^{1}\left(n_{1}, n_{2}\right) \\
& +\widehat{A}_{22} \delta z_{k+1}^{2}\left(n_{1}, n_{2}+1\right)+\widetilde{A}_{21} \delta z_{k+1}^{1}\left(n_{1}, n_{2}+1\right)+\widehat{A}_{22} \delta z_{k+1}^{2}\left(n_{1}, n_{2}+1\right)+B_{2}\left[u_{k+1}\left(n_{1}, n_{2}\right)-u_{k}\left(n_{1}, n_{2}\right)\right]
\end{aligned}
$$

where $n_{1} \in\left\{0,1, \ldots, N_{1}-1\right\}$ and $n_{2} \in\left\{0,1, \ldots, N_{2}-1\right\}$.

Then, using ILC law (8), we have

$$
\begin{aligned}
\delta z_{k+1}^{1}\left(n_{1}+1, n_{2}+1\right)= & \bar{A}_{11} \delta z_{k+1}^{1}\left(n_{1}+1, n_{2}\right)+\bar{A}_{12} \delta z_{k+1}^{2}\left(n_{1}+1, n_{2}\right)+\widehat{A}_{11} \delta z_{k+1}^{1}\left(n_{1}, n_{2}\right)+\widehat{A}_{12} \delta z_{k+1}^{2}\left(n_{1}, n_{2}\right) \\
& +\widetilde{A}_{11} \delta z_{k+1}^{1}\left(n_{1}, n_{2}+1\right)+\widetilde{A}_{12} \delta z_{k+1}^{2}\left(n_{1}, n_{2}+1\right)+B_{1} K e_{k}\left(n_{1}, n_{2}\right), \\
0= & \bar{A}_{21} \delta z_{k+1}^{1}\left(n_{1}+1, n_{2}\right)+\bar{A}_{22} \delta z_{k+1}^{2}\left(n_{1}+1, n_{2}\right)+\widehat{A}_{21} \delta z_{k+1}^{1}\left(n_{1}, n_{2}\right)+\widehat{A}_{22} \delta z_{k+1}^{2}\left(n_{1}, n_{2}\right) \\
& +\widetilde{A}_{21} \delta z_{k+1}^{1}\left(n_{1}, n_{2}+1\right)+\widetilde{A}_{22} \delta z_{k+1}^{2}\left(n_{1}, n_{2}+1\right)+B_{2} K e_{k}\left(n_{1}, n_{2}\right) .
\end{aligned}
$$

According to Assumption 2, premultiplying by $\widetilde{A}_{22}^{-1}$ on both sides of (15), we obtain

$$
\begin{aligned}
\delta z_{k+1}^{2}\left(n_{1}, n_{2}+1\right)= & -\widetilde{A}_{22}^{-1} \bar{A}_{21} \delta z_{k+1}^{1}\left(n_{1}+1, n_{2}\right)-\widetilde{A}_{22}^{-1} \bar{A}_{22} \delta z_{k+1}^{2}\left(n_{1}+1, n_{2}\right)-\widetilde{A}_{22}^{-1} \widehat{A}_{21} \delta z_{k+1}^{1}\left(n_{1}, n_{2}\right) \\
& -\widetilde{A}_{22}^{-1} \widehat{A}_{22} \delta z_{k+1}^{2}\left(n_{1}, n_{2}\right)-\widetilde{A}_{22}^{-1} \widetilde{A}_{21} \delta z_{k+1}^{1}\left(n_{1}, n_{2}+1\right)-\widetilde{A}_{22}^{-1} B_{2} K e_{k}\left(n_{1}, n_{2}\right) .
\end{aligned}
$$

Substituting (16) into (12), it yields

$$
\begin{aligned}
\delta z_{k+1}^{1}\left(n_{1}+1, n_{2}+1\right)= & \left(\bar{A}_{11}-\widetilde{A}_{12} \widetilde{A}_{22}^{-1} \bar{A}_{21}\right) \delta z_{k+1}^{1}\left(n_{1}+1, n_{2}\right)+\left(\bar{A}_{12}-\widetilde{A}_{12} \widetilde{A}_{22}^{-1} \bar{A}_{22}\right) \delta z_{k+1}^{2}\left(n_{1}+1, n_{2}\right) \\
& +\left(\widehat{A}_{11}-\widetilde{A}_{12} \widetilde{A}_{22}^{-1} \widehat{A}_{21}\right) \delta z_{k+1}^{1}\left(n_{1}, n_{2}\right)+\left(\widehat{A}_{12}-\widetilde{A}_{12} \widetilde{A}_{22}^{-1} \widehat{A}_{21}\right) \delta z_{k+1}^{2}\left(n_{1}, n_{2}\right) \\
& +\left(\widetilde{A}_{11}-\widetilde{A}_{12} \widetilde{A}_{22}^{-1} \widetilde{A}_{21}\right) \delta z_{k+1}^{1}\left(n_{1}, n_{2}+1\right)+\left(B_{1} K-\widetilde{A}_{12} \widetilde{A}_{22}^{-1} B_{2} K\right) e_{k}\left(n_{1}, n_{2}\right) .
\end{aligned}
$$

On the other hand, according to $e_{k}\left(n_{1}, n_{2}\right)=y_{d}\left(n_{1}, n_{2}\right)-y_{k}\left(n_{1}, n_{2}\right)$ and (6), it generates

$$
\begin{aligned}
e_{k+1}\left(n_{1}, n_{2}\right)-e_{k}\left(n_{1}, n_{2}\right) & =y_{d}\left(n_{1}, n_{2}\right)-y_{k+1}\left(n_{1}, n_{2}\right)-y_{d}\left(n_{1}, n_{2}\right)+y_{k}\left(n_{1}, n_{2}\right) \\
& =-C_{1}\left[z_{k+1}^{1}\left(n_{1}, n_{2}\right)-z_{k}^{1}\left(n_{1}, n_{2}\right)\right]-C_{2}\left[z_{k+1}^{2}\left(n_{1}, n_{2}\right)-z_{k}^{2}\left(n_{1}, n_{2}\right)\right]-D\left[u_{k+1}\left(n_{1}, n_{2}\right)-u_{k}\left(n_{1}, n_{2}\right)\right]
\end{aligned}
$$

where $n_{1} \in\left\{0,1, \ldots, N_{1}\right\}$ and $n_{2} \in\left\{0,1, \ldots, N_{2}\right\}$. From (11)-(12) and the ILC law (9), it yields

$$
e_{k+1}\left(n_{1}, n_{2}\right)=-C_{1} \delta z_{k+1}^{1}\left(n_{1}, n_{2}\right)-C_{2} \delta z_{k+1}^{2}\left(n_{1}, n_{2}\right)+\left(I_{s}-D K\right) e_{k}\left(n_{1}, n_{2}\right) .
$$


Let

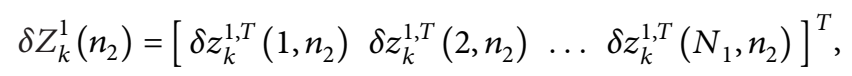

$$
\delta Z_{k}^{2}\left(n_{2}\right)=\left[\begin{array}{llll}
\delta z_{k}^{2, T}\left(1, n_{2}\right) & \delta z_{k}^{2, T}\left(2, n_{2}\right) & \ldots & \delta z_{k}^{2, T}\left(N_{1}, n_{2}\right)
\end{array}\right]^{T},
$$

$$
E_{k}\left(n_{2}\right)=\left[\begin{array}{llll}
e_{k}^{T}\left(0, n_{2}\right) & e_{k}^{T}\left(1, n_{2}\right) & \ldots & e_{k}^{T}\left(N_{1}, n_{2}\right)
\end{array}\right]^{T} .
$$

From (20)-(22), (17), (16), and (19) can be rewritten as

$$
\begin{aligned}
\Phi_{1} \delta Z_{k+1}^{1}\left(n_{2}+1\right)= & \Phi_{2} \delta Z_{k+1}^{1}\left(n_{2}\right)+\Phi_{3} \delta Z_{k+1}^{2}\left(n_{2}\right)+\Phi_{4} E_{k}\left(n_{2}\right)+\Phi_{5} \delta z_{k+1}^{1}\left(0, n_{2}+1\right) \\
& +\Phi_{6} \delta z_{k+1}^{1}\left(0, n_{2}\right)+\Phi_{7} \delta z_{k+1}^{2}\left(0, n_{2}\right), \\
\Psi_{0} \delta Z_{k+1}^{2}\left(n_{2}+1\right)= & \Psi_{1} \delta Z_{k+1}^{1}\left(n_{2}+1\right)+\Psi_{2} \delta Z_{k+1}^{1}\left(n_{2}\right)+\Psi_{3} \delta Z_{k+1}^{2}\left(n_{2}\right)+\Psi_{4} E_{k}\left(n_{2}\right) \\
& +\Psi_{5} \delta z_{k+1}^{1}\left(0, n_{2}+1\right)+\Psi_{6} \delta z_{k+1}^{1}\left(0, n_{2}\right)+\Psi_{7} \delta z_{k+1}^{2}\left(0, n_{2}\right)+\Psi_{8} \delta z_{k+1}^{2}\left(0, n_{2}+1\right), \\
E_{k+1}\left(n_{2}\right)= & \Theta_{1} \delta Z_{k+1}^{1}\left(n_{2}\right)+\Theta_{2} \delta Z_{k+1}^{2}\left(n_{2}\right)+\Theta_{3} E_{k}\left(n_{2}\right)+\Theta_{4} \delta z_{k+1}^{1}\left(0, n_{2}\right)+\Theta_{5} \delta z_{k+1}^{2}\left(0, n_{2}\right),
\end{aligned}
$$

where $\Phi_{i}, i \in\{1,2, \ldots, 7\}, \Psi_{j}, j \in\{0,1,2, \ldots, 8\}$, and $\Theta_{h}$, $h \in\{1,2, \ldots, 5\}$, are given in the next page. Since $\Phi_{1}$ is a

nonsingular block matrix, and premultiplying $\Phi_{1}^{-1}$ on both sides of (23), we get

$$
\begin{aligned}
\delta Z_{k+1}^{1}\left(n_{2}+1\right)= & \Phi_{1}^{-1} \Phi_{2} \delta Z_{k+1}^{1}\left(n_{2}\right)+\Phi_{1}^{-1} \Phi_{3} \delta Z_{k+1}^{2}\left(n_{2}\right)+\Phi_{1}^{-1} \Phi_{4} E_{k}\left(n_{2}\right)+\Phi_{1}^{-1} \Phi_{5} \delta z_{k+1}^{1}\left(0, n_{2}+1\right) \\
& +\Phi_{1}^{-1} \Phi_{6} \delta z_{k+1}^{1}\left(0, n_{2}\right)+\Phi_{1}^{-1} \Phi_{7} \delta z_{k+1}^{2}\left(0, n_{2}\right) .
\end{aligned}
$$

Substituting (26) into (24), we have

$$
\begin{aligned}
\Psi_{0} \delta Z_{k+1}^{2}\left(n_{2}+1\right)= & \left(\Psi_{1} \Phi_{1}^{-1} \Phi_{2}+\Psi_{2}\right) \delta Z_{k+1}^{1}\left(n_{2}\right)+\left(\Psi_{1} \Phi_{1}^{-1} \Phi_{3}+\Psi_{3}\right) \delta Z_{k+1}^{2}\left(n_{2}\right) \\
& +\left(\Psi_{1} \Phi_{1}^{-1} \Phi_{4}+\Psi_{4}\right) E_{k}\left(n_{2}\right)+\left(\Psi_{1} \Phi_{1}^{-1} \Phi_{5}+\Psi_{5}\right) \delta z_{k+1}^{1}\left(0, n_{2}+1\right) \\
& +\left(\Psi_{1} \Phi_{1}^{-1} \Phi_{6}+\Psi_{6}\right) \delta z_{k+1}^{1}\left(0, n_{2}\right)+\left(\Psi_{1} \Phi_{1}^{-1} \Phi_{7}+\Psi_{7}\right) \delta z_{k+1}^{2}\left(0, n_{2}\right)+\Psi_{8} \delta z_{k+1}^{2}\left(0, n_{2}+1\right) .
\end{aligned}
$$

Taking norms on both sides of (26), (27), and (25), respectively, and considering $\left\|\Psi_{0}\right\|=1$, there is

$$
\begin{aligned}
\left\|\delta Z_{k+1}^{1}\left(n_{2}+1\right)\right\| \leq & \left\|\Phi_{1}^{-1} \Phi_{2}\right\|\left\|\delta Z_{k+1}^{1}\left(n_{2}\right)\right\|+\left\|\Phi_{1}^{-1} \Phi_{3}\right\|\left\|\delta Z_{k+1}^{2}\left(n_{2}\right)\right\|+\left\|\Phi_{1}^{-1} \Phi_{4}\right\|\left\|E_{k}\left(n_{2}\right)\right\| \\
& +\left\|\Phi_{1}^{-1} \Phi_{5}\right\|\left\|\delta z_{k+1}^{1}\left(0, n_{2}+1\right)\right\|+\left\|\Phi_{1}^{-1} \Phi_{6}\right\|\left\|\delta z_{k+1}^{1}\left(0, n_{2}\right)\right\|+\left\|\Phi_{1}^{-1} \Phi_{7}\right\|\left\|\delta z_{k+1}^{2}\left(0, n_{2}\right)\right\|, \\
\left\|\delta Z_{k+1}^{2}\left(n_{2}+1\right)\right\| \leq & \left\|\Psi_{1} \Phi_{1}^{-1} \Phi_{2}+\Psi_{2}\right\|\left\|\delta Z_{k+1}^{1}\left(n_{2}\right)\right\|+\left\|\Psi_{1} \Phi_{1}^{-1} \Phi_{3}+\Psi_{3}\right\|\left\|\delta Z_{k+1}^{2}\left(n_{2}\right)\right\| \\
& +\left\|\Psi_{1} \Phi_{1}^{-1} \Phi_{4}+\Psi_{4}\right\|\left\|E_{k}\left(n_{2}\right)\right\|+\left\|\Psi_{1} \Phi_{1}^{-1} \Phi_{5}+\Psi_{5}\right\|\left\|\delta z_{k+1}^{1}\left(0, n_{2}+1\right)\right\| \\
& +\left\|\Psi_{1} \Phi_{1}^{-1} \Phi_{6}+\Psi_{6}\right\|\left\|\delta z_{k+1}^{1}\left(0, n_{2}\right)\right\|+\left\|\Psi_{1} \Phi_{1}^{-1} \Phi_{7}+\Psi_{7}\right\|\left\|\delta z_{k+1}^{2}\left(0, n_{2}\right)\right\|+\left\|\Psi_{8}\right\|\left\|\delta z_{k+1}^{2}\left(0, n_{2}+1\right)\right\|,
\end{aligned}
$$

$$
\begin{gathered}
\left\|E_{k+1}\left(n_{2}\right)\right\| \leq\left\|\Theta_{1}\right\|\left\|\delta Z_{k+1}^{1}\left(n_{2}\right)\right\|+\left\|\Theta_{2}\right\|\left\|\delta Z_{k+1}^{2}\left(n_{2}\right)\right\|+\left\|\Theta_{3}\right\|\left\|E_{k}\left(n_{2}\right)\right\|+\left\|\Theta_{4}\right\|\left\|\delta z_{k+1}^{1}\left(0, n_{2}\right)\right\| \\
+\left\|\Theta_{5}\right\|\left\|\delta z_{k+1}^{2}\left(0, n_{2}\right)\right\|,
\end{gathered}
$$


where

$$
\begin{aligned}
\Phi_{1} & =\left[\begin{array}{ccccc}
I_{r} & 0 & 0 & \cdots & 0 \\
-\widetilde{A}_{11}-\widetilde{A}_{12} \widetilde{A}_{22}^{-1} \widetilde{A}_{21} & I_{r} & 0 & \ldots & 0 \\
0 & -\widetilde{A}_{11}-\widetilde{A}_{12} \widetilde{A}_{22}^{-1} \widetilde{A}_{21} & I_{r} & \ddots & \vdots \\
\vdots & \ddots & \ddots & \ddots & 0 \\
0 & \ldots & 0 & -\widetilde{A}_{11}-\widetilde{A}_{12} \widetilde{A}_{22}^{-1} \widetilde{A}_{21} & I_{r}
\end{array}\right], \\
\Phi_{2} & =\left[\begin{array}{ccccc}
\bar{A}_{11}-\widetilde{A}_{12} \widetilde{A}_{22}^{-1} \bar{A}_{21} & 0 & 0 & \cdots & 0 \\
\widehat{A}_{12}-\widetilde{A}_{12} \widetilde{A}_{22}^{-1} \widehat{A}_{21} & \bar{A}_{11}-\widetilde{A}_{12} \widetilde{A}_{22}^{-1} \bar{A}_{21} & 0 & \ddots & 0 \\
0 & \widehat{A}_{12}-\widetilde{A}_{12} \widetilde{A}_{22}^{-1} \widehat{A}_{21} & \bar{A}_{11}-\widetilde{A}_{12} \widetilde{A}_{22}^{-1} \bar{A}_{21} & \ddots & \vdots \\
\vdots & \ddots & \ddots & \ddots & 0 \\
0 & \ldots & 0 & \widehat{A}_{12}-\widetilde{A}_{12} \widetilde{A}_{22}^{-1} \widehat{A}_{21} \bar{A}_{11}-\widetilde{A}_{12} \widetilde{A}_{22}^{-1} \bar{A}_{21}
\end{array}\right],
\end{aligned}
$$$$
\Phi_{3}=\left[\begin{array}{ccccc}
\bar{A}_{12}-\widetilde{A}_{12} \widetilde{A}_{22}^{-1} \bar{A}_{22} & 0 & 0 & \cdots & 0 \\
\widehat{A}_{12}-\widetilde{A}_{12} \widetilde{A}_{22}^{-1} \widehat{A}_{22} & \bar{A}_{12}-\widetilde{A}_{12} \widetilde{A}_{22}^{-1} \bar{A}_{22} & 0 & \ddots & 0 \\
0 & \widehat{A}_{12}-\widetilde{A}_{12} \widetilde{A}_{22}^{-1} \widehat{A}_{22} & \bar{A}_{12}-\widetilde{A}_{12} \widetilde{A}_{22}^{-1} \bar{A}_{22} & \ddots & \vdots \\
\vdots & \ddots & \ddots & \ddots & 0 \\
0 & \ldots & 0 & \widehat{A}_{12}-\widetilde{A}_{12} \widetilde{A}_{22}^{-1} \widehat{A}_{22} \bar{A}_{12}-\widetilde{A}_{12} \widetilde{A}_{22}^{-1} \bar{A}_{22}
\end{array}\right] \text {, }
$$$$
\Phi_{4}=\left[\begin{array}{cccc}
B_{1} K-\widetilde{A}_{12} \widetilde{A}_{22} B_{2} K & 0 & \ldots & 0 \\
0 & \ddots & \ddots & \vdots \\
\vdots & \ddots & B_{1} K-\widetilde{A}_{12} \widetilde{A}_{22} B_{2} K & 0 \\
0 & \ldots & 0 & 0
\end{array}\right] \text {, }
$$$$
\Phi_{5}=\left[\begin{array}{c}
\widetilde{A}_{11}-\widetilde{A}_{12} \widetilde{A}_{22}^{-1} \widetilde{A}_{21} \\
0 \\
\vdots \\
0
\end{array}\right] \text {, }
$$$$
\Phi_{6}=\left[\begin{array}{c}
\widehat{A}_{11}-\widetilde{A}_{12} \widetilde{A}_{22}^{-1} \widehat{A}_{21} \\
0 \\
\vdots \\
0
\end{array}\right] \text {, }
$$$$
\Phi_{7}=\left[\begin{array}{c}
\widehat{A}_{12}-\widetilde{A}_{12} \widetilde{A}_{22}^{-1} \widehat{A}_{22} \\
0 \\
\vdots \\
0
\end{array}\right] \text {, }
$$$$
\Psi_{0}=\left[\begin{array}{ccccc}
0 & 0 & 0 & \ldots & 0 \\
I_{p-r} & 0 & 0 & \ldots & 0 \\
0 & I_{p-r} & \ddots & \ddots & \vdots \\
\vdots & \ddots & \ddots & 0 & 0 \\
0 & \ldots & 0 & I_{p-r} & 0
\end{array}\right]
$$ 


$$
\begin{aligned}
& \Psi_{1}=\left[\begin{array}{ccccc}
0 & 0 & 0 & \ldots & 0 \\
-\widetilde{A}_{22}^{-1} \widetilde{A}_{21} & 0 & 0 & \ldots & 0 \\
0 & -\widetilde{A}_{22}^{-1} \widetilde{A}_{21} & 0 & \ddots & \vdots \\
\vdots & \ddots & \ddots & \ddots & 0 \\
0 & \ldots & 0 & -\widetilde{A}_{22}^{-1} \widetilde{A}_{21} & 0
\end{array}\right] \text {, } \\
& \Psi_{2}=\left[\begin{array}{ccccc}
-\widetilde{A}_{22}^{-1} \bar{A}_{21} & 0 & 0 & \ldots & 0 \\
-\widetilde{A}_{22}^{-1} \widehat{A}_{21} & -\widetilde{A}_{22}^{-1} \bar{A}_{21} & 0 & \ldots & 0 \\
0 & -\widetilde{A}_{22}^{-1} \widehat{A}_{21} & -\widetilde{A}_{22}^{-1} \bar{A}_{21} & \ddots & \vdots \\
\vdots & \ddots & \ddots & \ddots & 0 \\
0 & \ldots & 0 & -\widetilde{A}_{22}^{-1} \widehat{A}_{21}-\widetilde{A}_{22}^{-1} \bar{A}_{21}
\end{array}\right] \\
& \Psi_{3}=\left[\begin{array}{ccccc}
-\widetilde{A}_{22}^{-1} \bar{A}_{22} & 0 & 0 & \ldots & 0 \\
-\widetilde{A}_{22}^{-1} \widehat{A}_{22} & -\widetilde{A}_{22}^{-1} \bar{A}_{22} & 0 & \ldots & 0 \\
0 & -\widetilde{A}_{22}^{-1} \widehat{A}_{22} & -\widetilde{A}_{22}^{-1} \bar{A}_{22} & \ddots & \vdots \\
\vdots & \ddots & \ddots & \ddots & 0 \\
0 & \ldots & 0 & -\widetilde{A}_{22}^{-1} \widehat{A}_{22}-\widetilde{A}_{22}^{-1} \bar{A}_{22}
\end{array}\right] \text {, } \\
& \Psi_{4}=\left[\begin{array}{cccc}
-\widetilde{A}_{22}^{-1} B_{2} K & 0 & \ldots & 0 \\
0 & \ddots & \ddots & \vdots \\
\vdots & \ddots & -\widetilde{A}_{22}^{-1} B_{2} K & 0 \\
0 & \ldots & 0 & 0
\end{array}\right] \text {, } \\
& \Psi_{5}=\left[\begin{array}{c}
-\widetilde{A}_{22}^{-1} \widetilde{A}_{21} \\
0 \\
\vdots \\
0
\end{array}\right] \\
& \Psi_{6}=\left[\begin{array}{c}
-\widetilde{A}_{22}^{-1} \widehat{A}_{21} \\
0 \\
\vdots \\
0
\end{array}\right],
\end{aligned}
$$




$$
\begin{aligned}
& \Psi_{7}=\left[\begin{array}{c}
-\widetilde{A}_{22}^{-1} \widehat{A}_{22} \\
0 \\
\vdots \\
0
\end{array}\right] \\
& \Psi_{8}=\left[\begin{array}{c}
I_{p-r} \\
0 \\
\vdots \\
0
\end{array}\right] \\
& \Theta_{1}=\left[\begin{array}{ccccc}
0 & 0 & 0 & \ldots & 0 \\
-C_{1} & 0 & 0 & \ldots & 0 \\
0 & -C_{1} & 0 & \ddots & \vdots \\
\vdots & \ddots & \ddots & \ddots & 0 \\
0 & \ldots & 0 & -C_{1} & 0
\end{array}\right], \\
& \Theta_{2}=\left[\begin{array}{ccccc}
0 & 0 & 0 & \ldots & 0 \\
-C_{2} & 0 & 0 & \ddots & \vdots \\
0 & -C_{2} & 0 & \ddots & 0 \\
\vdots & \ddots & \ddots & \ddots & 0 \\
0 & \ldots & 0 & -C_{2} & 0
\end{array}\right] \text {, } \\
& \Theta_{3}=\left[\begin{array}{cccc}
I_{s}-D K & 0 & \cdots & 0 \\
0 & I_{s}-D K & \ddots & \vdots \\
\vdots & \ddots & \ddots & 0 \\
0 & \ldots & 0 & I_{s}-D K
\end{array}\right] \text {, } \\
& \Theta_{4}=\left[\begin{array}{c}
-C_{1} \\
0 \\
\vdots \\
0
\end{array}\right] \\
& \Theta_{5}=\left[\begin{array}{c}
-C_{2} \\
0 \\
\vdots \\
0
\end{array}\right]
\end{aligned}
$$


Letting $\delta Z_{k}\left(n_{2}\right)=\left[\left\|\delta Z_{k}^{1}\left(n_{2}\right)\right\|\left\|\delta Z_{k}^{2}\left(n_{2}\right)\right\|\right]^{T}$ and considering Definitions 1-2, (28)-(30) are reformulated as

$$
\begin{aligned}
\delta Z_{k+1}\left(n_{2}+1\right) \leq & \bar{\Phi}_{1} \delta Z_{k+1}\left(n_{2}\right)+\bar{\Phi}_{2}\left\|E_{k}\left(n_{2}\right)\right\|+\bar{\Phi}_{3}\left\|\delta z_{k+1}^{1}\left(0, n_{2}+1\right)\right\|+\bar{\Phi}_{4}\left\|\delta z_{k+1}^{1}\left(0, n_{2}\right)\right\| \\
& +\bar{\Phi}_{5}\left\|\delta z_{k+1}^{2}\left(0, n_{2}\right)\right\|+\bar{\Phi}_{6}\left\|\delta z_{k+1}^{2}\left(0, n_{2}+1\right)\right\|, \\
\left\|E_{k+1}\left(n_{2}\right)\right\| \leq & \bar{\Phi}_{7} \delta Z_{k+1}\left(n_{2}\right)+\bar{\Phi}_{8}\left\|E_{k}\left(n_{2}\right)\right\|+\bar{\Phi}_{9}\left\|\delta z_{k+1}^{1}\left(0, n_{2}\right)\right\|+\bar{\Phi}_{10}\left\|\delta z_{k+1}^{2}\left(0, n_{2}\right)\right\|
\end{aligned}
$$

where

$$
\begin{aligned}
& \bar{\Phi}_{1}=\left[\begin{array}{cc}
\left\|\Phi_{1}^{-1} \Phi_{2}\right\| & \left\|\Phi_{1}^{-1} \Phi_{3}\right\| \\
\left\|\Psi_{1} \Phi_{1}^{-1} \Phi_{2}+\Psi_{2}\right\|\left\|\Psi_{1} \Phi_{1}^{-1} \Phi_{3}+\Psi_{3}\right\|
\end{array}\right], \\
& \bar{\Phi}_{2}=\left[\begin{array}{c}
\left\|\Phi_{1}^{-1} \Phi_{4}\right\| \\
\left\|\Psi_{1} \Phi_{1}^{-1} \Phi_{4}+\Psi_{4}\right\|
\end{array}\right], \\
& \bar{\Phi}_{3}=\left[\begin{array}{c}
\left\|\Phi_{1}^{-1} \Phi_{5}\right\| \\
\left\|\Psi_{1} \Phi_{1}^{-1} \Phi_{5}+\Psi_{5}\right\|
\end{array}\right], \\
& \bar{\Phi}_{4}=\left[\begin{array}{c}
\left\|\Phi_{1}^{-1} \Phi_{6}\right\| \\
\left\|\Psi_{1} \Phi_{1}^{-1} \Phi_{6}+\Psi_{6}\right\|
\end{array}\right], \\
& \bar{\Phi}_{5}=\left[\begin{array}{c}
\left\|\Phi_{1}^{-1} \Phi_{7}\right\| \\
\left\|\Psi_{1} \Phi_{1}^{-1} \Phi_{7}+\Psi_{7}\right\|
\end{array}\right], \\
& \bar{\Phi}_{6}=\left[\begin{array}{c}
0 \\
\left\|\Psi_{8}\right\|
\end{array}\right], \\
& \bar{\Phi}_{7}=\left[\left\|\Theta_{1}\right\|\left\|\Theta_{2}\right\|\right], \\
& \bar{\Phi}_{8}=\left\|\Theta_{3}\right\|, \\
& \bar{\Phi}_{9}=\left\|\Theta_{4}\right\|, \\
& \bar{\Phi}_{10}=\left\|\Theta_{5}\right\| .
\end{aligned}
$$

$$
\begin{aligned}
\left\|\delta z_{k+1}^{1}\left(0, n_{2}\right)\right\| & =\left\|Q_{11}\left[x_{k+1}^{1}\left(0, n_{2}\right)-x_{k}^{1}\left(0, n_{2}\right)\right]+Q_{12}\left[x_{k+1}^{2}\left(0, n_{2}\right)-x_{k}^{2}\left(0, n_{2}\right)\right]\right\| \leq \bar{\Lambda}_{1}, \\
\left\|\delta z_{k+1}^{2}\left(0, n_{2}\right)\right\| & =\left\|Q_{21}\left[x_{k+1}^{1}\left(0, n_{2}\right)-x_{k}^{1}\left(0, n_{2}\right)\right]+Q_{22}\left[x_{k+1}^{2}\left(0, n_{2}\right)-x_{k}^{2}\left(0, n_{2}\right)\right]\right\| \leq \bar{\Lambda}_{2}, \\
\left\|\delta z_{k+1}^{1}\left(n_{1}, 0\right)\right\| & =\left\|Q_{11}\left[x_{k+1}^{1}\left(n_{1}, 0\right)-x_{k}^{1}\left(n_{1}, 0\right)\right]+Q_{12}\left[x_{k+1}^{2}\left(0, n_{2}\right)-x_{k}^{2}\left(0, n_{2}\right)\right]\right\| \leq \bar{\Lambda}_{3}, \\
\left\|\delta z_{k+1}^{2}\left(n_{1}, 0\right)\right\| & =\left\|Q_{21}\left[x_{k+1}^{1}\left(n_{1}, 0\right)-x_{k}^{1}\left(n_{1}, 0\right)\right]+Q_{22}\left[x_{k+1}^{2}\left(n_{1}, 0\right)-x_{k}^{2}\left(n_{1}, 0\right)\right]\right\| \leq \bar{\Lambda}_{4}, \\
\left\|\delta Z_{k+1}^{1}(0)\right\| & \left\|\left[\begin{array}{c}
\delta z_{k+1}^{1}(1,0) \\
\delta z_{k+1}^{1}(2,0) \\
\vdots \\
\delta z_{k+1}^{1}\left(N_{1}, 0\right)
\end{array}\right]\right\| \leq \bar{\Lambda}_{5}, \\
\left\|\delta Z_{k+1}^{2}(0)\right\|= & \left\|\left[\begin{array}{c}
\delta z_{k+1}^{2}(1,0) \\
\delta z_{k+1}^{2}(2,0) \\
\vdots \\
\delta z_{k+1}^{2}\left(N_{1}, 0\right)
\end{array}\right]\right\| \leq \bar{\Lambda}_{6},
\end{aligned}
$$


where $\bar{\Lambda}_{1}, \bar{\Lambda}_{2}, \bar{\Lambda}_{3}, \bar{\Lambda}_{4}, \bar{\Lambda}_{5}$, and $\bar{\Lambda}_{6}$ are dependent on bound parameters $\Lambda_{1}$ and $\Lambda_{2}$ in Assumption 1. From (35) and (36), we know that $\delta Z_{k+1}(0)$ is bounded. In addition, $\left\|E_{0}\left(n_{2}\right)\right\|$ is bounded for $n_{2} \in\left\{0,1, \ldots, N_{2}-1\right\}$ due to the boundedness property of $y_{d}\left(n_{1}, n_{2}\right)$ and $y_{0}\left(n_{1}, n_{2}\right)$. Using Lemma 2 in [22], if $\bar{\Phi}_{8}<1$ (equivalently, $\left\|I_{s}-D K\right\|<1$ ), there is

$$
\limsup _{k \longrightarrow+\infty} \delta Z_{k+1}\left(n_{2}\right) \leq \beta_{\delta Z}, \quad n_{2} \in\left\{0,1, \ldots, N_{2}\right\}
$$$$
\limsup _{k \rightarrow+\infty}\left\|E_{k}\left(n_{2}\right)\right\| \leq \beta_{E}, \quad n_{2} \in\left\{0,1, \ldots, N_{2}-1\right\} .
$$

In addition, taking $n_{2}=N_{2}$ in (19), we have

$$
e_{k+1}\left(n_{1}, N_{2}\right)=-C_{1} \delta z_{k+1}^{1}\left(n_{1}, N_{2}\right)-C_{2} \delta z_{k+1}^{2}\left(n_{1}, N_{2}\right)+\left(I_{s}-D K\right) e_{k}\left(n_{1}, N_{2}\right)
$$

where $n_{1} \in\left\{0,1, \ldots, N_{1}\right\}$. Taking norm on both sides of (39), we have

$$
\left\|e_{k+1}\left(n_{1}, N_{2}\right)\right\| \leq\left\|C_{1}\right\|\left\|\delta z_{k+1}^{1}\left(n_{1}, N_{2}\right)\right\|+\left\|C_{2}\right\|\left\|\delta z_{k+1}^{2}\left(n_{1}, N_{2}\right)\right\|+\left\|I_{s}-D K\right\|\left\|e_{k}\left(n_{1}, N_{2}\right)\right\| .
$$

From (37), we know $\left\|\delta z_{k+1}^{1}\left(n_{1}, N_{2}\right)\right\|$ and $\left\|\delta z_{k+1}^{2}\left(n_{1}, N_{2}\right)\right\|$ are bounded. Using Lemma 1, if $\left\|I_{s}-D K\right\|<1$ is satisfied, there is

$$
\underset{k \longrightarrow+\infty}{\limsup }\left\|e_{k}\left(n_{1}, N_{2}\right)\right\| \leq b_{e}, \quad n_{1} \in\left\{0,1, \ldots, N_{1}\right\} .
$$

From (38), (41), and the definition on $E_{k}\left(n_{2}\right)$ in (22), it yields

$$
\limsup _{k \longrightarrow+\infty}\left\|e_{k}\left(n_{1}, n_{2}\right)\right\| \leq \beta_{e}
$$

where $n_{1} \in\left\{0,1, \ldots, N_{1}\right\}$ and $n_{2} \in\left\{0,1, \ldots, N_{2}\right\}$. Theorem 1 is completed.

As iteration-invariant boundary states are imposed on 2D LDSFM (1), there is Corollary 1.
Corollary 1. For 2-D LDSFM (1) under Assumption 1 with $\Lambda_{1}=0$ and $\Lambda_{2}=0$ and Assumptions 2-3, use P-type ILC law (9). If there exists the learning gain $K$ satisfying $\left\|I_{s}-D K\right\|<1$, then, the tracking error $e_{k}\left(n_{1}, n_{2}\right)$ converges to zero, i.e.,

$$
\lim _{k \longrightarrow+\infty} e_{k}\left(n_{1}, n_{2}\right)=0
$$

where $n_{1} \in\left\{0,1, \ldots, N_{1}\right\}$ and $n_{2} \in\left\{0,1, \ldots, N_{2}\right\}$.

\section{Extension to 2-D LDSFM with $D=0$}

In this section, under Assumptions 1-3, we investigate the ILC issue for 2-D LDSFM with $D=0$ [28], which is described in the following:

$$
\left\{\begin{array}{l}
E_{g} x_{k}\left(n_{1}+1, n_{2}+1\right)=A_{1} x_{k}\left(n_{1}+1, n_{2}\right)+A_{2} x_{k}\left(n_{1}, n_{2}\right)+A_{3}\left(n_{1}, n_{2}+1\right)+B u_{k}\left(n_{1}, n_{2}\right), \\
y_{k}\left(n_{1}, n_{2}\right)=C x_{k}\left(n_{1}, n_{2}\right),
\end{array}\right.
$$

where $E_{g}, A_{1}, A_{2}, A_{3}, B$, and $C$ have been described in (1).

Based on Assumptions 1-3, we will discuss the robustness and convergence property of ILC law (45) for 2-D LDSFM (44). Theorem 2 is given as follows.

Theorem 2. For 2-D LDSFM (44) under Assumptions 1-3, the following P-type ILC law for $n_{1} \in\left\{0,1, \ldots, N_{1}-1\right\}$ and $n_{2} \in\left\{0,1, \ldots, N_{2}-1\right\}$,

$$
u_{k+1}\left(n_{1}, n_{2}\right)=u_{k}\left(n_{1}, n_{2}\right)+K e_{k}\left(n_{1}+1, n_{2}+1\right),
$$

is applied. If there exists the learning gain $K$ satisfying

$$
\bar{\Phi}_{8}<1
$$

where $\quad \bar{\Phi}_{8}=\left\|I_{s N_{1}}+\widehat{\Theta}_{1} \Phi_{1}^{-1} \Phi_{4}\right\|+\left\|\widehat{\Theta}_{2}\right\|\left\|\Psi_{1} \Phi_{1}^{-1} \Phi_{4}+\Psi_{4}\right\|$, where $\widehat{\Theta}_{1}, \widehat{\Theta}_{2}, \Phi_{1}, \Phi_{4}, \Psi_{1}$, and $\Psi_{4}$ are given in (52)-(55); the tracking error $e_{k}\left(n_{1}, n_{2}\right)$, with $n_{1} \in\left\{1,2, \ldots, N_{1}\right\}$ and $n_{2} \in\left\{1,2, \ldots, N_{2}\right\}$ converges to a bounded range, i.e.,

$$
\limsup _{k \rightarrow+\infty}\left\|e_{k}\left(n_{1}, n_{2}\right)\right\| \leq \beta_{e}^{\prime} \text {, }
$$

where $\beta_{e}^{\prime}$ is a certain bound relevant with the bound parameters $\Lambda_{1}$ and $\Lambda_{2}$ in Assumption 1.

Proof. For $n_{1} \in\left\{0,1, \ldots, N_{1}\right\}$ and $n_{2} \in\left\{0,1, \ldots, N_{2}\right\}$, let $\delta z_{k+1}^{1}\left(n_{1}, n_{2}\right)$ and $\delta z_{k+1}^{2}\left(n_{1}, n_{2}\right)$ be defined as (11) and (12). Using singular value decomposition theory on (43) and following the same procedure as (17) and (16), there is 


$$
\begin{aligned}
\delta z_{k+1}^{1}\left(n_{1}+1, n_{2}+1\right)= & \left(\bar{A}_{11}-\widetilde{A}_{12} \widetilde{A}_{22}^{-1} \bar{A}_{21}\right) \delta z_{k+1}^{1}\left(n_{1}+1, n_{2}\right)+\left(\bar{A}_{12}-\widetilde{A}_{12} \widetilde{A}_{22}^{-1} \bar{A}_{22}\right) \delta z_{k+1}^{2}\left(n_{1}+1, n_{2}\right) \\
& +\left(\widehat{A}_{11}-\widetilde{A}_{12} \widetilde{A}_{22}^{-1} \widehat{A}_{21}\right) \delta z_{k+1}^{1}\left(n_{1}, n_{2}\right)+\left(\widehat{A}_{12}-\widetilde{A}_{12} \widetilde{A}_{22}^{-1} \widehat{A}_{21}\right) \delta z_{k+1}^{2}\left(n_{1}, n_{2}\right) \\
& +\left(\widetilde{A}_{11}-\widetilde{A}_{12} \widetilde{A}_{22}^{-1} \widetilde{A}_{21}\right) \delta z_{k+1}^{1}\left(n_{1}, n_{2}+1\right)+\left(B_{1} K-\widetilde{A}_{12} \widetilde{A}_{22}^{-1} B_{2} K\right) e_{k}\left(n_{1}+1, n_{2}+1\right), \\
\delta z_{k+1}^{2}\left(n_{1}, n_{2}+1\right)= & -\widetilde{A}_{22}^{-1} \bar{A}_{21} \delta z_{k+1}^{1}\left(n_{1}+1, n_{2}\right)-\widetilde{A}_{22}^{-1} \bar{A}_{22} \delta z_{k+1}^{2}\left(n_{1}+1, n_{2}\right)-\widetilde{A}_{22}^{-1} \widehat{A}_{21} \delta z_{k+1}^{1}\left(n_{1}, n_{2}\right) \\
& -\widetilde{A}_{22}^{-1} \widehat{A}_{22} \delta z_{k+1}^{2}\left(n_{1}, n_{2}\right)-\widetilde{A}_{22}^{-1} \widetilde{A}_{21} \delta z_{k+1}^{1}\left(n_{1}, n_{2}+1\right)-\widetilde{A}_{22}^{-1} B_{2} K e_{k}\left(n_{1}+1, n_{2}+1\right) .
\end{aligned}
$$

On the other hand, according to

$e_{k}\left(n_{1}, n_{2}\right)=y_{d}\left(n_{1}, n_{2}\right)-y_{k}\left(n_{1}, n_{2}\right)$ and $(44)$, it generates

$$
\begin{aligned}
& e_{k+1}\left(n_{1}+1, n_{2}+1\right)-e_{k}\left(n_{1}+1, n_{2}+1\right)=y_{d}\left(n_{1}+1, n_{2}+1\right)-y_{k+1}\left(n_{1}+1, n_{2}+1\right)-y_{d}\left(n_{1}+1, n_{2}+1\right) \\
& \quad+y_{k}\left(n_{1}+1, n_{2}+1\right)=-C_{1}\left[z_{k+1}^{1}\left(n_{1}+1, n_{2}+1\right)-z_{k}^{1}\left(n_{1}+1, n_{2}+1\right)\right]-C_{2}\left[z_{k+1}^{2}\left(n_{1}+1, n_{2}+1\right)-z_{k}^{2}\left(n_{1}+1, n_{2}+1\right)\right],
\end{aligned}
$$

where $n_{1} \in\left\{0,1, \ldots, N_{1}-1\right\}$ and $n_{2} \in\left\{0,1, \ldots, N_{2}-1\right\}$.

From (11)-(12), it becomes

$$
e_{k+1}\left(n_{1}+1, n_{2}+1\right)-e_{k}\left(n_{1}+1, n_{2}+1\right)=-C_{1} \delta z_{k+1}^{1}\left(n_{1}+1, n_{2}+1\right)-C_{2} \delta z_{k+1}^{2}\left(n_{1}+1, n_{2}+1\right) .
$$

Let

$$
\widehat{E}_{k}\left(n_{2}\right)=\left[\begin{array}{llll}
e_{k}^{T}\left(1, n_{2}\right) & e_{k}^{T}\left(2, n_{2}\right) & \ldots & e_{k}^{T}\left(N_{1}, n_{2}\right)
\end{array}\right]^{T}
$$

From (51), (50) can be reformulated as

$\widehat{E}_{k+1}\left(n_{2}+1\right)=\widehat{\Theta}_{1} \delta Z_{k+1}^{1}\left(n_{2}+1\right)+\widehat{\Theta}_{2} \delta Z_{k+1}^{2}\left(n_{2}+1\right)+\widehat{E}_{k}\left(n_{2}+1\right)$,

where $\delta Z_{k+1}^{1}\left(n_{2}\right)$ and $\delta Z_{k+1}^{2}\left(n_{2}\right)$ are described in (20) and (21). $\widehat{\Theta}_{1}$ and $\widehat{\Theta}_{2}$ are given as

$$
\begin{aligned}
& \widehat{\Theta}_{1}=\left[\begin{array}{cccc}
-C_{1} & 0 & \ldots & 0 \\
0 & -C_{1} & \ddots & \vdots \\
\vdots & \ddots & \ddots & 0 \\
0 & \cdots & 0 & -C_{1}
\end{array}\right], \\
& \widehat{\Theta}_{2}=\left[\begin{array}{cccc}
-C_{2} & 0 & \cdots & 0 \\
0 & -C_{2} & \ddots & \vdots \\
\vdots & \ddots & \ddots & 0 \\
0 & \ldots & 0 & -C_{2}
\end{array}\right] .
\end{aligned}
$$

$$
\begin{aligned}
\delta Z_{k+1}^{1}\left(n_{2}+1\right)= & \Phi_{1}^{-1} \Phi_{2} \delta Z_{k+1}^{1}\left(n_{2}\right)+\Phi_{1}^{-1} \Phi_{3} \delta Z_{k+1}^{2}\left(n_{2}\right)+\Phi_{1}^{-1} \Phi_{4} \widehat{E}_{k}\left(n_{2}+1\right)+\Phi_{1}^{-1} \Phi_{5} \delta z_{k+1}^{1}\left(0, n_{2}+1\right) \\
& +\Phi_{1}^{-1} \Phi_{6} \delta z_{k+1}^{1}\left(0, n_{2}\right)+\Phi_{1}^{-1} \Phi_{7} \delta z_{k+1}^{2}\left(0, n_{2}\right), \\
\Psi_{0} \delta Z_{k+1}^{2}\left(n_{2}+1\right)= & \left(\Psi_{1} \Phi_{1}^{-1} \Phi_{2}+\Psi_{2}\right) \delta Z_{k+1}^{1}\left(n_{2}\right)+\left(\Psi_{1} \Phi_{1}^{-1} \Phi_{3}+\Psi_{3}\right) \delta Z_{k+1}^{2}\left(n_{2}\right) \\
& +\left(\Psi_{1} \Phi_{1}^{-1} \Phi_{4}+\Psi_{4}\right) \widehat{E}_{k}\left(n_{2}+1\right)+\left(\Psi_{1} \Phi_{1}^{-1} \Phi_{5}+\Psi_{5}\right) \delta z_{k+1}^{1}\left(0, n_{2}+1\right) \\
& +\left(\Psi_{1} \Phi_{1}^{-1} \Phi_{6}+\Psi_{6}\right) \delta z_{k+1}^{1}\left(0, n_{2}\right)+\left(\Psi_{1} \Phi_{1}^{-1} \Phi_{7}+\Psi_{7}\right) \delta z_{k+1}^{2}\left(0, n_{2}\right)+\Psi_{8} \delta z_{k+1}^{2}\left(0, n_{2}+1\right),
\end{aligned}
$$


where $\Phi_{i}, i \in\{1,2,3,5,6,7\}$, and $\Psi_{j}, j \in\{0,1,2,3,5,6,7,8\}$, have been described in (23) and (24); $\Phi_{4}$ and $\Psi_{4}$ are given as

$$
\begin{aligned}
& \Phi_{4}=\left[\begin{array}{cccc}
B_{1} K-\widetilde{A}_{12} \widetilde{A}_{22}^{-1} B_{2} K & 0 & \cdots & 0 \\
0 & B_{1} K-\widetilde{A}_{12} \widetilde{A}_{22}^{-1} B_{2} K & \ddots & \vdots \\
\vdots & \ddots & \ddots & 0 \\
0 & \ldots & 0 & B_{1} K-\widetilde{A}_{12} \widetilde{A}_{22}^{-1} B_{2} K
\end{array}\right] \text {, } \\
& \Psi_{4}=\left[\begin{array}{cccc}
-\widetilde{A}_{22}^{-1} B_{2} K & 0 & \cdots & 0 \\
0 & -\widetilde{A}_{22}^{-1} B_{2} K & \ddots & \vdots \\
\vdots & \ddots & \ddots & 0 \\
0 & \cdots & 0 & -\widetilde{A}_{22}^{-1} B_{2} K
\end{array}\right] \text {. }
\end{aligned}
$$

Substituting (54) into (52), we have

$$
\begin{aligned}
\widehat{E}_{k+1}\left(n_{2}+1\right)= & \widehat{\Theta}_{1} \Phi_{1}^{-1} \Phi_{2} \delta Z_{k+1}^{1}\left(n_{2}\right)+\widehat{\Theta}_{1} \Phi_{1}^{-1} \Phi_{3} \delta Z_{k+1}^{2}\left(n_{2}\right)+\left(I_{s N_{1}}+\widehat{\Theta}_{1} \Phi_{1}^{-1} \Phi_{4}\right) \widehat{E}_{k}\left(n_{2}+1\right) \\
& +\widehat{\Theta}_{2} \delta Z_{k+1}^{2}\left(n_{2}+1\right)+\widehat{\Theta}_{1} \Phi_{1}^{-1} \Phi_{5} \delta z_{k+1}^{1}\left(0, n_{2}+1\right)+\widehat{\Theta}_{1} \Phi_{1}^{-1} \Phi_{6} \delta z_{k+1}^{1}\left(0, n_{2}\right)+\widehat{\Theta}_{1} \Phi_{1}^{-1} \Phi_{7} \delta z_{k+1}^{2}\left(0, n_{2}\right) .
\end{aligned}
$$

Taking norms on both sides of (54)-(55), respectively, and considering $\left\|\Psi_{0}\right\|=1$, there is

$$
\begin{aligned}
\left\|\delta Z_{k+1}^{1}\left(n_{2}+1\right)\right\| \leq & \left\|\Phi_{1}^{-1} \Phi_{2}\right\|\left\|\delta Z_{k+1}^{1}\left(n_{2}\right)\right\|+\left\|\Phi_{1}^{-1} \Phi_{3}\right\|\left\|\delta Z_{k+1}^{2}\left(n_{2}\right)\right\|+\left\|\Phi_{1}^{-1} \Phi_{4}\right\|\left\|\widehat{E}_{k}\left(n_{2}+1\right)\right\| \\
& +\left\|\Phi_{1}^{-1} \Phi_{5}\right\|\left\|\delta z_{k+1}^{1}\left(0, n_{2}+1\right)\right\|+\left\|\Phi_{1}^{-1} \Phi_{6}\right\|\left\|\delta z_{k+1}^{1}\left(0, n_{2}\right)\right\|+\left\|\Phi_{1}^{-1} \Phi_{7}\right\|\left\|\delta z_{k+1}^{2}\left(0, n_{2}\right)\right\|, \\
\left\|\delta Z_{k+1}^{2}\left(n_{2}+1\right)\right\| \leq & \left\|\Psi_{1} \Phi_{1}^{-1} \Phi_{2}+\Psi_{2}\right\|\left\|\delta Z_{k+1}^{1}\left(n_{2}\right)\right\|+\left\|\Psi_{1} \Phi_{1}^{-1} \Phi_{3}+\Psi_{3}\right\|\left\|\delta Z_{k+1}^{2}\left(n_{2}\right)\right\| \\
& +\left\|\Psi_{1} \Phi_{1}^{-1} \Phi_{4}+\Psi_{4}\right\|\left\|\widehat{E}_{k}\left(n_{2}+1\right)\right\|+\left\|\Psi_{1} \Phi_{1}^{-1} \Phi_{5}+\Psi_{5}\right\|\left\|\delta z_{k+1}^{1}\left(0, n_{2}+1\right)\right\| \\
& +\left\|\Psi_{1} \Phi_{1}^{-1} \Phi_{6}+\Psi_{6}\right\|\left\|\delta z_{k+1}^{1}\left(0, n_{2}\right)\right\|+\left\|\Psi_{1} \Phi_{1}^{-1} \Phi_{7}+\Psi_{7}\right\|\left\|\delta z_{k+1}^{2}\left(0, n_{2}\right)\right\|+\left\|\Psi_{8}\right\|\left\|\delta z_{k+1}^{2}\left(0, n_{2}+1\right)\right\| .
\end{aligned}
$$

Taking norms on both sides of (57), and considering (59), it yields

$$
\begin{aligned}
\left\|\widehat{E}_{k+1}\left(n_{2}+1\right)\right\| \leq & \left(\left\|\widehat{\Theta}_{1} \Phi_{1}^{-1} \Phi_{2}\right\|+\left\|\widehat{\Theta}_{2}\right\|\left\|\Psi_{1} \Phi_{1}^{-1} \Phi_{2}+\Psi_{2}\right\|\right)\left\|\delta Z_{k+1}^{1}\left(n_{2}\right)\right\| \\
& +\left(\left\|\widehat{\Theta}_{1} \Phi_{1}^{-1} \Phi_{3}\right\|+\left\|\widehat{\Theta}_{2}\right\|\left\|\Psi_{1} \Phi_{1}^{-1} \Phi_{2}+\Psi_{3}\right\|\right)\left\|\delta Z_{k+1}^{2}\left(n_{2}\right)\right\|+\left(\left\|I_{s N_{1}}+\widehat{\Theta}_{1} \Phi_{1}^{-1} \Phi_{4}\right\|+\left\|\widehat{\Theta}_{2}\right\|\left\|\Psi_{1} \Phi_{1}^{-1} \Phi_{4}+\Psi_{4}\right\|\right)\left\|\widehat{E}_{k}\left(n_{2}+1\right)\right\| \\
& +\left(\left\|\widehat{\Theta}_{1} \Phi_{1}^{-1} \Phi_{5}\right\|+\left\|\widehat{\Theta}_{2}\right\|\left\|\Psi_{1} \Phi_{1}^{-1} \Phi_{5}+\Psi_{5}\right\|\right)\left\|\delta z_{k+1}^{1}\left(0, n_{2}+1\right)\right\|+\left(\left\|\widehat{\Theta}_{1} \Phi_{1}^{-1} \Phi_{6}\right\|+\left\|\widehat{\Theta}_{2}\right\|\left\|\Psi_{1} \Phi_{1}^{-1} \Phi_{6}+\Psi_{6}\right\|\right)\left\|\delta z_{k+1}^{1}\left(0, n_{2}\right)\right\| \\
& +\left(\left\|\widehat{\Theta}_{1} \Phi_{1}^{-1} \Phi_{7}\right\|+\left\|\widehat{\Theta}_{2}\right\|\left\|\Psi_{1} \Phi_{1}^{-1} \Phi_{7}+\Psi_{7}\right\|\right)\left\|\delta z_{k+1}^{2}\left(0, n_{2}\right)\right\|+\left\|\widehat{\Theta}_{2}\right\|\left\|\Psi_{8}\right\|\left\|\delta z_{k+1}^{2}\left(0, n_{2}+1\right)\right\| .
\end{aligned}
$$


Define $\quad \delta Z_{k}\left(n_{2}\right)=\left[\left\|\delta Z_{k}^{1}\left(n_{2}\right)\right\|\left\|\delta Z_{k}^{2}\left(n_{2}\right)\right\|\right]^{T}, \quad$ and (58)-(60) can be rewritten as

$$
\begin{aligned}
\delta Z_{k+1}\left(n_{2}+1\right) \leq & \bar{\Phi}_{1} \delta Z_{k+1}\left(n_{2}\right)+\bar{\Phi}_{2}\left\|E_{k}\left(n_{2}+1\right)\right\|+\bar{\Phi}_{3}\left\|\delta z_{k+1}^{1}\left(0, n_{2}+1\right)\right\|+\bar{\Phi}_{4}\left\|\delta z_{k+1}^{1}\left(0, n_{2}\right)\right\| \\
& +\bar{\Phi}_{5}\left\|\delta z_{k+1}^{2}\left(0, n_{2}\right)\right\|+\bar{\Phi}_{6}\left\|\delta z_{k+1}^{2}\left(0, n_{2}+1\right)\right\|, \\
\left\|\widehat{E}_{k+1}\left(n_{2}+1\right)\right\| \leq & \bar{\Phi}_{7} \delta Z_{k+1}\left(n_{2}\right)+\bar{\Phi}_{8}\left\|\widehat{E}_{k}\left(n_{2}+1\right)\right\|+\bar{\Phi}_{9}\left\|\delta z_{k+1}^{1}\left(0, n_{2}+1\right)\right\|+\bar{\Phi}_{10}\left\|\delta z_{k+1}^{1}\left(0, n_{2}\right)\right\| \\
& +\bar{\Phi}_{11}\left\|\delta z_{k+1}^{2}\left(0, n_{2}\right)\right\|+\bar{\Phi}_{12}\left\|\delta z_{k+1}^{2}\left(0, n_{2}+1\right)\right\|,
\end{aligned}
$$

where $\bar{\Phi}_{i}, i \in\{1,2, \ldots, 6\}$, are given in (33)-(34); $\bar{\Phi}_{j}$, $j \in\{7,8, \ldots, 12\}$, are shown as

$$
\begin{aligned}
& \bar{\Phi}_{7}=\left[\left\|\widehat{\Theta}_{1} \Phi_{1}^{-1} \Phi_{2}\right\|+\left\|\widehat{\Theta}_{2}\right\|\left\|\Psi_{1} \Phi_{1}^{-1} \Phi_{2}+\Psi_{2}\right\|\left\|\widehat{\Theta}_{1} \Phi_{1}^{-1} \Phi_{3}\right\|+\left\|\widehat{\Theta}_{2}\right\|\left\|\Psi_{1} \Phi_{1}^{-1} \Phi_{3}+\Psi_{3}\right\|\right], \\
& \bar{\Phi}_{8}=\left\|I_{s N_{1}}+\widehat{\Theta}_{1} \Phi_{1}^{-1} \Phi_{4}\right\|+\left\|\widehat{\Theta}_{2}\right\|\left\|\Psi_{1} \Phi_{1}^{-1} \Phi_{4}+\Psi_{4}\right\|, \\
& \bar{\Phi}_{9}=\left\|\widehat{\Theta}_{1} \Phi_{1}^{-1} \Phi_{5}\right\|+\left\|\widehat{\Theta}_{2}\right\|\left\|\Psi_{1} \Phi_{1}^{-1} \Phi_{5}+\Psi_{5}\right\|, \\
& \bar{\Phi}_{10}=\left\|\widehat{\Theta}_{1} \Phi_{1}^{-1} \Phi_{6}\right\|+\left\|\widehat{\Theta}_{2}\right\|\left\|\Psi_{1} \Phi_{1}^{-1} \Phi_{6}+\Psi_{6}\right\|, \\
& \bar{\Phi}_{11}=\left\|\widehat{\Theta}_{1} \Phi_{1}^{-1} \Phi_{7}\right\|+\left\|\widehat{\Theta}_{2}\right\|\left\|\Psi_{1} \Phi_{1}^{-1} \Phi_{7}+\Psi_{7}\right\|, \\
& \bar{\Phi}_{12}=\left\|\widehat{\Theta}_{2}\right\|\left\|\Psi_{8}\right\| .
\end{aligned}
$$

From Assumption 1, we obtain $\left\|\delta z_{k+1}^{1}\left(0, n_{2}\right)\right\|$, $\left\|\delta z_{k+1}^{2}\left(0, n_{2}\right)\right\|$, and $\delta Z_{k+1}(0)$ are bounded. Using Lemma 2 in [22], if $\Phi_{8}<1$, there is

$$
\underset{k \longrightarrow+\infty}{\limsup }\left\|\widehat{E}_{k}\left(n_{2}+1\right)\right\| \leq \beta_{\widehat{E}}, \quad n_{2} \in\left\{0,1, \ldots, N_{2}-1\right\} .
$$

From (51), we obtain

$$
\underset{k \longrightarrow+\infty}{\limsup }\left\|e_{k}\left(n_{1}, n_{2}\right)\right\| \leq \beta_{e}^{\prime}
$$

where $n_{1} \in\left\{1,2, \ldots, N_{1}\right\}$ and $n_{2} \in\left\{1,2, \ldots, N_{2}\right\}$. Theorem 2 is completed.

Corollary 2. For 2-D LDSFM (44) under Assumption 1 with $\Lambda_{1}=0$ and $\Lambda_{2}=0$ and Assumptions 2-3, use the P-type ILC law (45). If there exists the learning gain $K$ satisfying (46), then the tracking error $e_{k}\left(n_{1}, n_{2}\right)$ converges to zero, i.e.,

$$
\lim _{k \longrightarrow+\infty} e_{k}\left(n_{1}, n_{2}\right)=0 \text {, }
$$

where $n_{1} \in\left\{1,2, \ldots, N_{1}\right\}$ and $n_{2} \in\left\{1,2, \ldots, N_{2}\right\}$.

Remark 2. It is noted that 2-D LDSFM contains a 2-D linear discrete singular Attasi model as a special case. Also, a 2-D singular Roesser model under some specified coefficient matrix requirements can be converted into 2-D LDSFM [32]. Consequently, depending on the ILC results obtained from 2-D LDSFM, it is easy to extend the 2-D singular Roesser model and singular Attasi model.

\section{Illustrative Examples}

In this section, to demonstrate the feasibility and effectiveness of the designed ILC algorithm for 2-D LDSFM, computer simulation is conducted for mathematical systems.

Example 1. Consider the ILC issue for 2-D LDSFM (44) with the following parameters:

$$
\begin{aligned}
E_{g} & =\left[\begin{array}{ll}
1 & 1 \\
1 & 1
\end{array}\right], \\
A_{1} & =\left[\begin{array}{ll}
0.1 & 0.01 \\
0.01 & 0.03
\end{array}\right], \\
A_{2} & =\left[\begin{array}{ll}
0.1 & 0.02 \\
0.01 & 0.04
\end{array}\right], \\
A_{3} & =\left[\begin{array}{ll}
0.01 & 0.02 \\
0.1 & 0.3
\end{array}\right], \\
B & =\left[\begin{array}{ll}
1 \\
1
\end{array}\right], \\
C & =\left[\begin{array}{ll}
-1 & -0.5
\end{array}\right] .
\end{aligned}
$$

Taking $N_{1}=N_{2}=20$, the desired surface trajectory $y_{d}\left(n_{1}, n_{2}\right)$ is described by $y_{d}\left(n_{1}, n_{2}\right)=\sin \left[0.2 \pi\left(n_{1}+n_{2}\right)\right]$ for $n_{1} \in\{0,1, \ldots, 20\}$ and $n_{2} \in\{0,1, \ldots, 20\}$, which is shown in Figure 1. We select two nonsingular transformation matrices $P=\left[\begin{array}{cc}0.5 & 0.5 \\ 0.2 & -0.2\end{array}\right]$ and $Q=\left[\begin{array}{cc}0.5 & 0.1 \\ 0.5 & -0.1\end{array}\right]$. In ILC law 


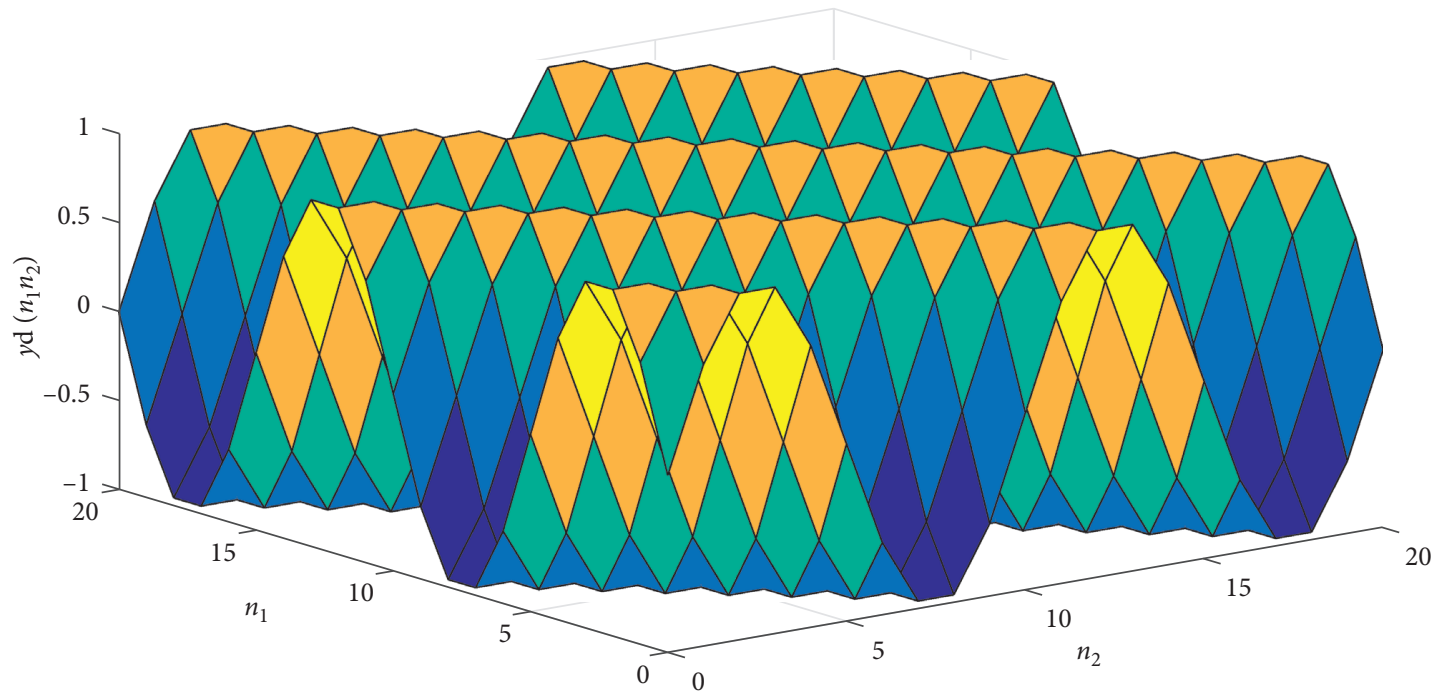

Figure 1: The desired surface trajectory $y_{d}\left(n_{1}, n_{2}\right)$ for $n_{1} \in\{0,1, \ldots, 20\}$ and $n_{2} \in\{0,1, \ldots, 20\}$.

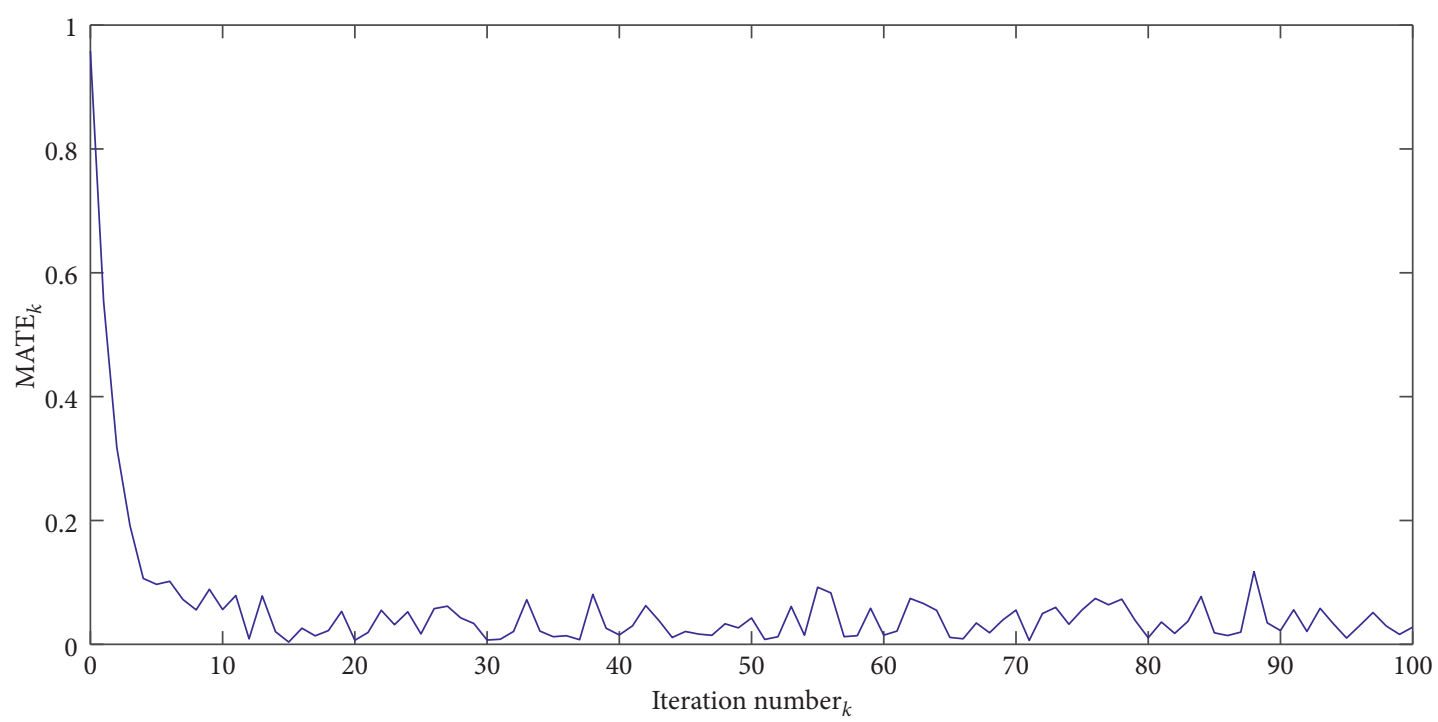

FIgURE 2: Under the ILC law (38), the profile of tracking error index MATE $_{k}$ with iteration number $k$.

(45), let the initial control input be given as $u_{0}\left(n_{1}, n_{2}\right)=0$, where $n_{1} \in\{0,1, \ldots, 19\}$ and $n_{2} \in\{0,1, \ldots, 19\}$, and the learning gain $K$ be selected as $K=-0.4$, which satisfies convergence condition (46) in Theorem 2 and Corollary 2. The maximum absolute tracking error index $\mathrm{MATE}_{k}$ is adopted to evaluate the ILC tracking performance, which is given as follows:

$$
\operatorname{MATE}_{k}=\max _{n_{1} \in\{1,2, \ldots, 20\}} \max _{n_{2} \in\{1,2, \ldots, 20\}}\left|y_{d}\left(n_{1}, n_{2}\right)-y_{k}\left(n_{1}, n_{2}\right)\right| .
$$

Case 1. Corresponding to Assumption 1 with iterationvarying boundary states, let the boundary states $x_{k}\left(0, n_{2}\right)$, $n_{2} \in\{1,2, \ldots, 20\}$, and $x_{k}\left(n_{1}, 0\right), n_{1} \in\{0,1, \ldots, 20\}$, be given as

$$
\begin{aligned}
& x_{k}\left(0, n_{2}\right)=\left[\begin{array}{c}
0 \\
\sin \left(0.2 \pi n_{2}\right)
\end{array}\right]+\left[\begin{array}{l}
m_{1, k} \\
m_{2, k}
\end{array}\right], \\
& x_{k}\left(n_{1}, 0\right)=\left[\begin{array}{c}
-0.5 \sin \left(0.2 \pi n_{1}\right) \\
0
\end{array}\right]+\left[\begin{array}{l}
n_{1, k} \\
n_{2, k}
\end{array}\right],
\end{aligned}
$$

where $m_{1, k}, m_{2, k}, n_{1, k}$, and $n_{2, k}$ vary randomly at $(-0.5,0.5)$, $(-0.1,0.1),(-0.5,0.5)$, and $(-0.1,0.1)$ along the iteration $k$. Figure 2 displays the profile of $\mathrm{MATE}_{k}$ with iteration number $k$ by using ILC law (45). Obviously, robust boundedness of ultimate ILC tracking error is validated from Figure 2.

Case 2. Corresponding to Assumption 1 with $\Lambda_{1}=0$ and $\Lambda_{2}=0$, let the boundary states satisfy 

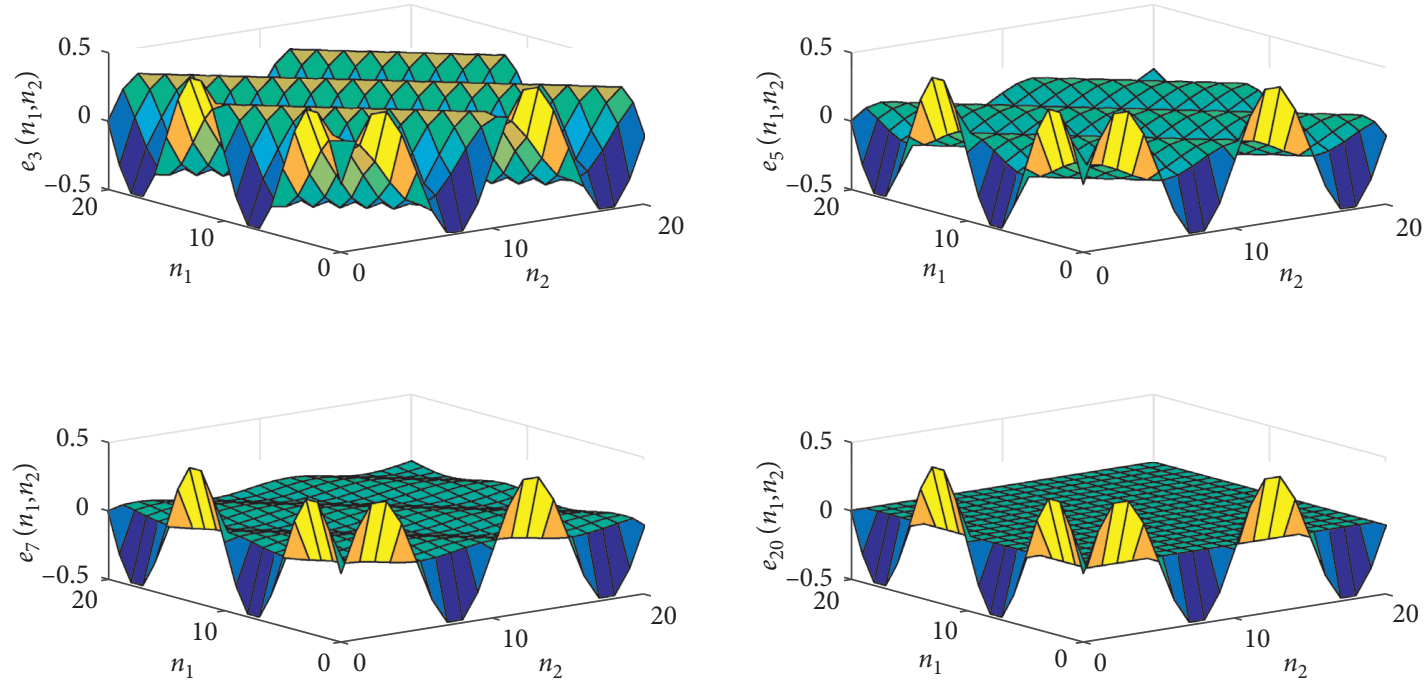

FiguRE 3: Under ILC law (45), the tracking error surface $e_{k}\left(n_{1}, n_{2}\right)$ at $k=3,5,7,20$.

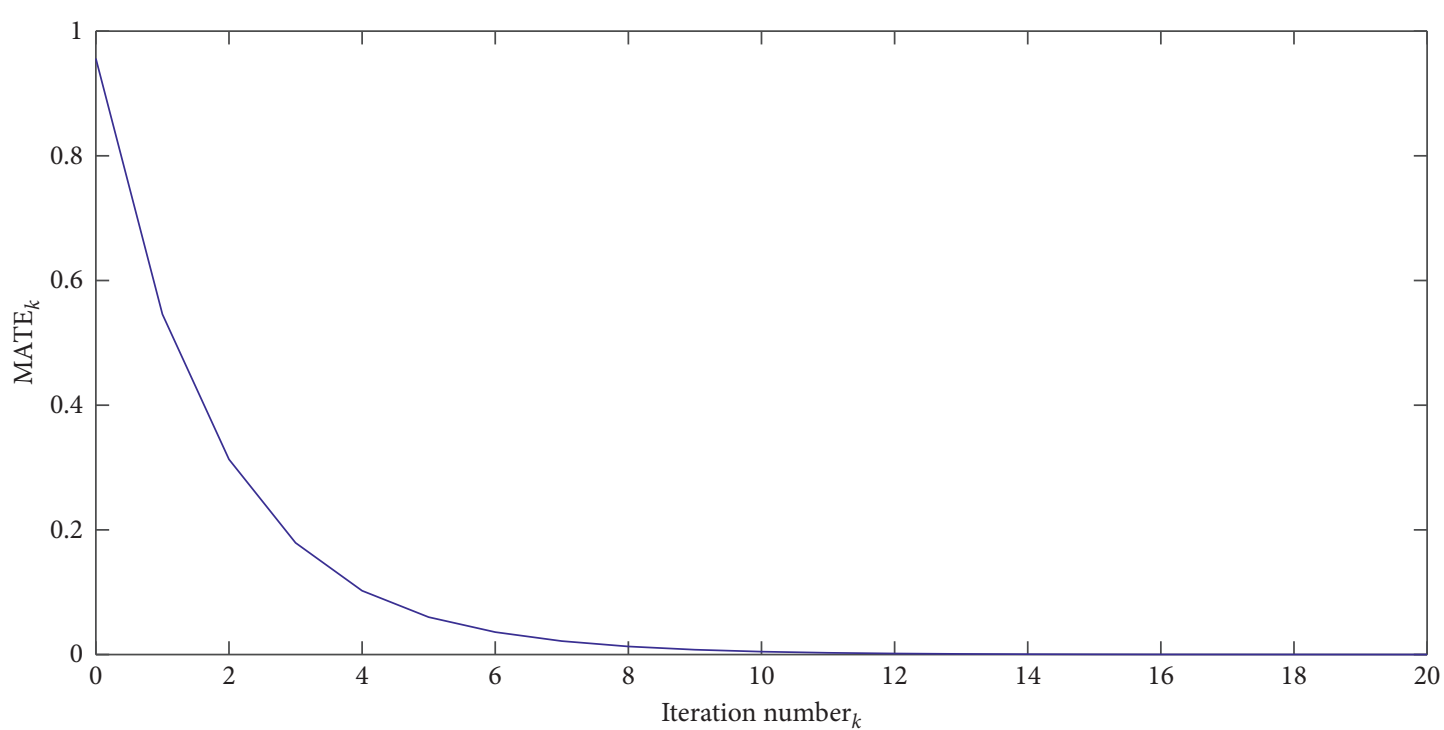

Figure 4: Under ILC law (45), the profile of tracking error index $\mathrm{MATE}_{k}$ with iteration number $k$.

$$
\begin{aligned}
& x_{k}\left(0, n_{2}\right)=\left[\begin{array}{ll}
0 & \sin \left(0.2 \pi n_{2}\right)
\end{array}\right]^{T}, \quad n_{2} \in\{1,2, \ldots, 20\} \\
& x_{k}\left(n_{1}, 0\right)=\left[-0.5 \sin \left(0.2 \pi n_{1}\right) \quad 0\right]^{T}, \quad n_{1} \in\{0,1, \ldots, 20\} .
\end{aligned}
$$

Figure 3 displays the profile of $\mathrm{MATE}_{k}$ with iteration number $k$ by using ILC law (45). The tracking error surface $e_{k}\left(n_{1}, n_{2}\right)$ at $k=3,5,7,20$ is shown in Figure 4. Apparently, a perfect tracking to repetitive reference trajectory $y_{d}\left(n_{1}, n_{2}\right)$ except the boundaries $n_{1}=0$ and $n_{2}=0$ can be observed from Figures 3-4, and the effectiveness of the presented ILC algorithm is illustrated.

\section{Conclusions}

At present, the ILC tracking issue for 2-D LDSFM under iteration-varying boundary states has not been studied. This paper first introduces singular value decomposition theory into ILC investigations on 2-D LDSFM. In addition, 3-D linear inequality stability theory is first proposed to analysis the convergence property on ILC algorithms, which is a novel analysis method. In the future work, more robust ILC uncertainties for 2-D nonlinear singular systems will be considered.

\section{Data Availability}

All the data used to support the study have been included within the article.

\section{Conflicts of Interest}

The authors declare no conflicts of interest.

\section{Acknowledgments}

This work was supported in part by the National Natural Science Foundation of China under Grant 61573385, the Professorial and Doctoral Scientific Research Foundation of 
Huizhou University under Grants 2020JB017 and 2020JB011, Natural Science Foundation of Guangdong Province under Grant 2018A030313711, Huizhou Science and Technology Plan Project under Grant 2020SD0406034, the Characteristic Innovation Projects of Universities in Guangdong Province under Grant 2018KTSCX215, and Program for Innovative Research Team of Huizhou University, IRTHZU.

\section{References}

[1] Y. Zou and S. L. Campbell, "The jump behavior and stability analysis for 2-D singular systems," Multidimensional Systems and Signal Processing, vol. 11, no. 4, pp. 321-338, 2000.

[2] C. Cai, W. Wang, and Y. Zou, "A note on the internal stability for 2-D singular discrete systems," Multidimensional Systems and Signal Processing, vol. 15, no. 2, pp. 197-204, 2004.

[3] T. Kaczorek, "Acceptable input sequences for singular 2-D linear systems," IEEE Transactions on Automatic Control, vol. 38, no. 9, pp. 1391-1394, 1993.

[4] H. Xu, Y. Zou, S. Xu, and J. Lam, "Bounded real lemma and robust control of 2-D singular Roesser models," Systems \& Control Letters, vol. 54, no. 4, pp. 339-346, 2005.

[5] W. Q. Wang and Y. Zou, "The detectability and observer design of 2-D singular systems," IEEE Transactions on Circuits and Systems-I: Fundamental Theory and Applications, vol. 49, no. 5, pp. 698-703, 2002.

[6] H. Xu and Y. Zou, "control for 2-D singular delayed systems," International Journal of Systems Science, vol. 42, no. 4, pp. 609-619, 2011.

[7] M. S. Boudellioua, K. Galkowski, and E. Rogers, "Equivalence of wave linear repetitive processes and the singular 2-D Roesser state-space model," Multidimensional Systems and Signal Processing, vol. 31, no. 1, pp. 103-116, 2020.

[8] E. Rimon and A. Stappen, "Immobilizing 2-D serial chains in form-closure grasps," IEEE Transactions on Robotics, vol. 28, no. 1, pp. 32-43, 2012.

[9] Z. Wei, M. J. Wang, Y. J. Cai, and L. Wang, "Milling force prediction for ball-end milling of 3D curved surfaces," Journal of Mechanical Engineering, vol. 49, no. 1, pp. 178-184, 2013.

[10] D. A. Bristow, M. Tharayil, and A. G. Alleyne, "A survey of iterative learning control," IEEE Control Systems Magazine, vol. 26, no. 3, pp. 96-114, 2006.

[11] J.-X. Xu, "A survey on iterative learning control for nonlinear systems," International Journal of Control, vol. 84, no. 7, pp. 1275-1294, 2011.

[12] D. Shen and X. Li, "A survey on iterative learning control with randomly varying trial lengths: model, synthesis, and convergence analysis," Annual Reviews in Control, vol. 48, pp. 89-102, 2019.

[13] M. Yu and S. Chai, "A survey on high-order internal model based iterative learning control," IEEE Access, vol. 7, pp. 127024-127031, 2019.

[14] D. Meng, "Convergence conditions for solving robust iterative learning control problems under nonrepetitive model uncertainties," IEEE Transactions on Neural Networks and Learning Systems, vol. 30, no. 6, pp. 1908-1919, 2019.

[15] X. Bu and $\mathrm{Z}$. Hou, "Adaptive iterative learning control for linear systems with binary-valued observations," IEEE Transactions on Neural Networks and Learning Systems, vol. 29, no. 1, pp. 232-237, 2018.

[16] R. Chi, Y. Hui, B. Huang, and Z. Hou, “Adjacent-agent dynamic linearization-based iterative learning formation control," IEEE Transactions on Cybernetics, vol. 50, no. 10, p. $4358,2020$.

[17] H. Afkhami, A. Argha, M. Roopaei, and M. A. Nouri, "Optimal iterative learning control method for 2-D systems using 1-D model (WAM) of 2-D systems," World Applied Science Journal, vol. 13, no. 11, pp. 2410-2419, 2011.

[18] M. Roopaei, A. Argha, and P. Karimaghaee, "On the control of 2-D systems by intelligent schemes," World Applied Science Journal, vol. 6, no. 3, pp. 385-392, 2009.

[19] T. F. Xiao and X. D. Li, "PID-type iterative learning control for 2-D Roesser model," in, in Proceedings of the IEEE 6th Data Driven Control and Learning Systems, pp. 400-404, Chongqing, China, May 2017.

[20] K. Wan and X.-D. Li, "Iterative learning control for twodimensional linear discrete systems with Fornasini-Marchesini model," International Journal of Control, Automation and Systems, vol. 15, no. 4, pp. 1710-1719, 2017.

[21] K. Wan and X. D. Li, "High-order internal model based iterative learning control for 2-D linear FMMI systems with iteration-varying trajectory tracking," IEEE Transactions on Systems, Man, Cybernetics: Systems, vol. 51, no. 3, pp. 14621472, 2021.

[22] K. Wan and X. D. Li, "Robust iterative learning control of 2-D linear discrete FMMII systems subject to iteration-dependent uncertainties," IEEE Transactions on Systems, Man, Cybernetics: Systems, 2019.

[23] K. Wan, D. Xu, and Y.-s. Wei, "Iterative learning control for 2-D linear discrete Fornasini-Marchesini model with input saturation," International Journal of Systems Science, vol. 51, no. 8, pp. 1482-1494, 2020.

[24] Q.-Y. Xu, X.-D. Li, and M.-M. Lv, “Adaptive ILC for tracking non-repetitive reference trajectory of 2-D FMM under random boundary condition," International Journal of Control, Automation and Systems, vol. 14, no. 2, pp. 478-485, 2016.

[25] P. P. Gu, T. S. Tian, and Q. Liu, "Iterative learning control for a class of discrete-time singular systems," Advances in Difference Equations, vol. 1, pp. 1-14, 2018.

[26] P. P. Gu, T. S. Tian, and Q. Liu, "Iterative learning control for switched singular time-delay systems," Journal of Vibration and Control, vol. 24, no. 20, pp. 4839-4849, 2018.

[27] Q. Liu, S. Tian, and P. Gu, "P-type iterative learning control algorithm for a class of linear singular impulsive systems," Journal of the Franklin Institute, vol. 355, no. 9, pp. 39263937, 2018.

[28] Y. Zou and H. Xu, "Duality of 2-D singular systems of Roesser models," Journal of Control Theory and Applications, vol. 5, no. 1, pp. 37-41, 2007.

[29] S. Y. Xu and L. James, Robust Control and Filtering of Singular Systems, Springer-Verlag, Berlin, Germany, 2006.

[30] A. Benzaouia, A. Hmamed, and F. Tadeo, Two-dimensional Systems, Springer Publishing Company, Berlin, Germany, 2015.

[31] J. Gregor, "On the singular Fornasini-Marchesini model of 2D discrete systems," IEEE Transactions on Automatic Control, vol. 37, no. 5, pp. 636-639, 1992.

[32] T. Kaczorek, Two-dimensional Linear Systems, SpringerVerlag, Berlin, Germany, 1985. 